\title{
Sector magnets or transverse electromagnetic fields in cylindrical coordinates
}

\author{
T. Zolkin \\ Fermilab, P.O. Box 500, Batavia, Illinois 60510-5011, USA
}

(Received 9 March 2016; published 10 April 2017)

\begin{abstract}
Laplace's equation is considered for scalar and vector potentials describing electric or magnetic fields in cylindrical coordinates, with invariance along the azimuthal coordinate. A series of special functions are found which, when expanded to lowest order in power series in radial and vertical coordinates, replicate harmonic polynomials in two variables. These functions are based on radial harmonics found by Edwin M. McMillan forty years ago. In addition to McMillan's harmonics, a second family of radial harmonics is introduced to provide a symmetric description between electric and magnetic fields and to describe fields and potentials in terms of the same functions. Formulas are provided which relate any transverse fields specified by the coefficients in the power series expansion in radial or vertical planes in cylindrical coordinates with the set of new functions. This result is important for potential theory and for theoretical study, design and proper modeling of sector dipoles, combined function dipoles and any general sector element for accelerator physics. All results are presented in connection with these problems.
\end{abstract}

DOI: 10.1103/PhysRevAccelBeams.20.043501

\section{INTRODUCTION}

The description of sector magnets, any curved magnet symmetric along its azimuthal cylindrical coordinate (longitudinal coordinate in accelerator physics) is an important issue. Every modern accelerator code includes such elements, the most important being combined function dipoles. A widely used method, which goes back to Karl Brown's 1968 paper [1], is based on a solution of Laplace's equation for a scalar potential using a power series in cylindrical coordinates. A similar approach applied to Laplace's equation for the longitudinal component of a vector potential can be found for example in [2]. The same approach appears in more recent books, e.g. in great detail in [3].

Two major bottlenecks should be noticed. First, if one looks for a solution in the form of a series, then these series must be truncated. In our case truncation means that potentials no longer satisfy Laplace's equation. (Of course potentials can "satisfy" Laplace's equation up to any desired order by keeping more and more terms in the expansion.) More importantly, the recurrence equation is undetermined. In every new order of recurrence one has to assign an arbitrary constant, which will affect all other higher order terms. This ambiguity leads to the fact that there is no preferred, unique choice of basis functions; it makes it difficult to compare accelerator codes, since

\footnotetext{
*zolkin@fnal.gov
}

Published by the American Physical Society under the terms of the Creative Commons Attribution 4.0 International license. Further distribution of this work must maintain attribution to the author(s) and the published article's title, journal citation, and DOI. different assumptions might be used for representations of basis functions.

This indeterminacy has a simple geometrical illustration. Looking for a field with a pure normal dipole component on a circular equilibrium orbit in lowest order, one can come up with an almost arbitrary shape of the magnet's north pole if its south pole is symmetric with respect to the midplane. In the case of a dipole, the series can be truncated by keeping only its dipole component. For higher order multipoles in cylindrical coordinates truncation without violation of Laplace's equation is not possible.

While working on an implementation of sector magnets for Synergia, I found assumptions which let me sum series for pure electric and magnetic skew and normal multipoles. Looking further for symmetry in the description allowed me to generate a family of solutions in which all the series could be summed, so that no truncation was required. While discussing my results with Sergei Nagaitsev, he brought my attention to an article by McMillan written in 1975 [4]. As I found later, the same result was independently obtained by Mane and published in the same journal about 20 years later [5] without citing McMillan's original work. It made me want to write this article in order to bring attention back to these forgotten results.

Joining my results to McMillan's, I would like to present a new representation for multipole expansions in cylindrical coordinates. Any transverse field can be expanded in terms of these functions and related to power series expansions in horizontal or vertical planes. The new approach does not contradict previous results but embraces them. The ambiguity in choice of coefficients and the problem of truncation are resolved. Thus it can be employed for theoretical studies, design and simulation of sector magnets. 
The paper is structured as follows. Section II provides the expansion of fields in multipoles for cases with zero and constant curvatures. The connection between this approach and Taylor expansion is provided at the end of this section. Appendices A and B describe global and curvilinear Frenet-Serret coordinate systems including general equations of motion for a particle in it. The case of pure transverse electric or magnetic fields is described in Secs. B 1 and B 2. Appendix C contains the main differential relations used in this article. Finally, Appendixes D-F are supplementary materials with harmonics, fields, potentials and Taylor series.

\section{EXPANSION OF TRANSVERSE ELECTROMAGNETIC FIELDS}

In appendixes A and B we provided dynamical equations of motion without specifying how to represent electromagnetic fields. In the next two subsections we will discuss the multipole field expansion for the two most important types of elements: R-element for $\kappa=0$ and S-element defined for $\kappa=$ const $=1 / R_{0}$, see Fig. 1 .

"R-" stands for "rectangular." This element is the one with $\left(q_{1}, q_{2}, q_{3}\right)$ simply being a right-handed Cartesian coordinate system, which we will denote as $(x, y, z)$. Fields in such elements are invariant along the $z$ axis, and the elements usually serve as quadrupoles, sextupoles, octupoles or combined function correctors. In addition one can design pure R-dipoles, while combined function bending

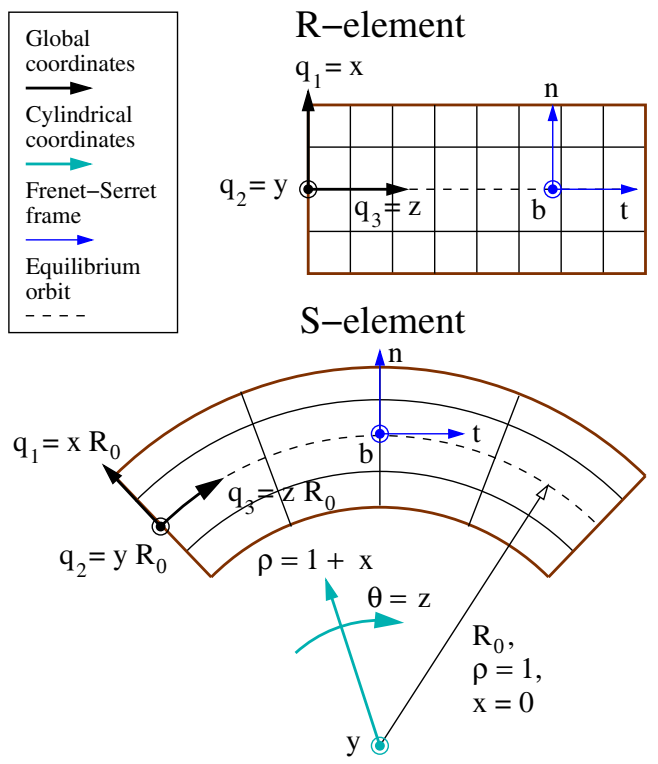

FIG. 1. Illustration of R- and S-elements. Elements are shown in brown. Global curvilinear coordinates with associated grid lines are shown in black. Black dashed line represents an equilibrium orbit. An example of the Frenet-Serret frame attached to an equilibrium orbit is drawn in blue colors. For S-element, an additional right-handed normalized cylindrical system is added and shown in cyan. magnets are exotic and very complicated since the equilibrium orbit will not coincide with the axis of symmetry.

S-element is the element defined with the natural sector coordinate system. Defining the set of normalized coordinates $\left(x=q_{1} / R_{0}, y=q_{2} / R_{0}, z=q_{3} / R_{0}\right)$, one can see that it simply can be related to normalized right-handed cylindrical coordinates $\left(\rho=1+x, y, \theta=z / R_{0}\right)$ and thus all fields are invariant along azimuthal coordinate $\theta$. S-elements are suitable for the design of combined function bending magnets, since in contrast to R-elements, the equilibrium orbit follows along $\theta$.

\section{A. Multipoles in Cartesian coordinates}

In Cartesian coordinates, Laplace's equations for transverse electro- and magnetostatic fields are in the same form which significantly simplifies the problem:

$$
\begin{aligned}
& \Delta_{\perp} \Phi=\frac{\partial^{2} \Phi}{\partial x^{2}}+\frac{\partial^{2} \Phi}{\partial y^{2}}=0, \\
& \Delta_{\perp} \mathbf{A}=\left(\frac{\partial^{2} A_{z}}{\partial x^{2}}+\frac{\partial^{2} A_{z}}{\partial y^{2}}\right) \hat{\mathbf{e}}_{z}=0 .
\end{aligned}
$$

Introduction of complex variables allows a very compact description of this problem with a unified description of electric and magnetic fields [6]. Suppose we have a holomorphic function of complex variable $\mathcal{Z}=x+i y$, which we will call a complex scalar potential, whose real part is defined to be the longitudinal component of a vector potential and whose imaginary part is the electric scalar potential,

$$
\Omega(\mathcal{Z})=A_{z}(x, y)+i \Phi(x, y) .
$$

Since the real and imaginary parts of any holomorphic function are harmonic functions, $A_{z}$ and $\Phi$ automatically satisfy Laplace's equation. Indeed, suppose we have a vector field $\mathbf{F}=\left(F_{x}, F_{y}\right)$. Introducing the Wirtinger derivatives,

$$
\frac{\partial}{\partial \mathcal{Z}}=\frac{1}{2}\left(\frac{\partial}{\partial x}-i \frac{\partial}{\partial y}\right) \text { and } \quad \frac{\partial}{\partial \overline{\mathcal{Z}}}=\frac{1}{2}\left(\frac{\partial}{\partial x}+i \frac{\partial}{\partial y}\right)
$$

one can write

$$
\begin{aligned}
& \frac{\partial \Omega}{\partial \overline{\mathcal{Z}}}=0, \\
& \frac{\partial \Omega}{\partial \mathcal{Z}}=F(\mathcal{Z}),
\end{aligned}
$$

where the first equation is the Cauchy-Riemann condition for $\Omega$, which guarantees that this field can be implemented via either magnetic or electric potentials:

$$
\begin{aligned}
& F_{x}=-\frac{\partial \Phi}{\partial x}=\frac{\partial A_{z}}{\partial y}, \\
& F_{y}=-\frac{\partial \Phi}{\partial y}=-\frac{\partial A_{z}}{\partial x} .
\end{aligned}
$$


TABLE I. Formulas for the scalar potential, longitudinal component of the vector potential and field components for pure normal and skew $2 n$-poles in Cartesian coordinates.

\begin{tabular}{lc}
\hline \hline Normal & Skew \\
\hline $\bar{\Phi}^{(n)}=-\bar{C}_{n} \frac{\mathcal{B}_{n}}{n !}$ & $\underline{\Phi}^{(n)}=-\underline{C}_{n} \frac{\mathcal{A}_{n}}{n !}$ \\
$\bar{A}_{z}^{(n)}=-\bar{C}_{n} \frac{\mathcal{A}_{n}}{n !}$ & $\underline{A}_{z}^{(n)}=\underline{C}_{n} \frac{\mathcal{B}_{n}}{n !}$ \\
$\bar{F}_{\mathrm{x}}^{(n)}=\bar{C}_{n} \frac{\mathcal{B}_{n-1}}{(n-1) !}$ & $\underline{F}_{\mathrm{x}}^{(n)}=\underline{C}_{n} \frac{\mathcal{A}_{n-1}}{(n-1) !}$ \\
$\bar{F}_{\mathrm{y}}^{(n)}=\bar{C}_{n} \frac{\mathcal{A}_{n-1}}{(n-1) !}$ & $\underline{F}_{\mathrm{y}}^{(n)}=-\underline{C}_{n} \frac{\mathcal{B}_{n-1}}{(n-1) !}$ \\
\hline \hline
\end{tabular}

The second equation defines the complex function of field components such that

$$
F_{x}=-\mathfrak{s} F(\mathcal{Z}) \text { and } F_{y}=-\mathfrak{R} F(\mathcal{Z}),
$$

which all together are equivalent to $\mathbf{F}=-\nabla \Phi=\nabla \times \mathbf{A}$. The complex function $F(\mathcal{Z})$ is the holomorphic function again and the Cauchy-Riemann equation gives

$$
\frac{\partial F}{\partial \overline{\mathcal{Z}}}=0
$$

which asserts that field $\mathbf{F}$ is irrotational and divergence free which is equivalent to time-independent free of electric charge and current density Maxwell's equations

$$
\nabla \cdot \mathbf{F}=0 \text { and } \nabla \times \mathbf{F}=0 .
$$

For accelerator physics purposes the expansion of fields usually represented in terms of homogeneous harmonic polynomials of two variables, which are defined through the complex power function,

$$
\begin{aligned}
\mathcal{A}_{n}(x, y)=\mathfrak{i} \mathcal{Z}^{n} & =\frac{1}{2}\left[(x+i y)^{n}+(x-i y)^{n}\right] \\
& =\sum_{k=0}^{n}\left(\begin{array}{l}
n \\
k
\end{array}\right) x^{n-k} y^{k} \cos \frac{k \pi}{2}, \\
\mathcal{B}_{n}(x, y)=\Im \mathcal{Z}^{n} & =\frac{1}{2 i}\left[(x+i y)^{n}-(x-i y)^{n}\right] \\
& =\sum_{k=0}^{n}\left(\begin{array}{l}
n \\
k
\end{array}\right) x^{n-k} y^{k} \sin \frac{k \pi}{2} .
\end{aligned}
$$

Explicit expressions are well known and up to tenth order are listed in Table VII of Appendix D. These functions satisfy the transverse Laplace's equation and are related to each other through the Cauchy-Riemann equation as

$$
\frac{\partial \mathcal{A}_{n}}{\partial x}=\frac{\partial \mathcal{B}_{n}}{\partial y} \quad \text { and } \quad \frac{\partial \mathcal{A}_{n}}{\partial y}=-\frac{\partial \mathcal{B}_{n}}{\partial x} .
$$

In addition one can introduce "ladder-like" lowering and raising integro-differential operators which relate functions of different order to each other:

$$
n(\mathcal{A}, \mathcal{B})_{n-1}=\frac{\partial}{\partial x}(\mathcal{A}, \mathcal{B})_{n}= \pm \frac{\partial}{\partial y}(\mathcal{B}, \mathcal{A})_{n}
$$

and

$$
(\mathcal{A}, \mathcal{B})_{n}=n \int_{0}^{x} \mathrm{~d} x(\mathcal{A}, \mathcal{B})_{n-1}+y^{n}(\cos , \sin ) \frac{n \pi}{2}
$$

with $\mathcal{A}_{0}=1$ and $\mathcal{B}_{0}=0$.

Thus one can define two independent sets of solutions: normal (sometimes called upright or straight) and skew pure multipoles, which we will denote with overline $\overline{(\ldots)}$
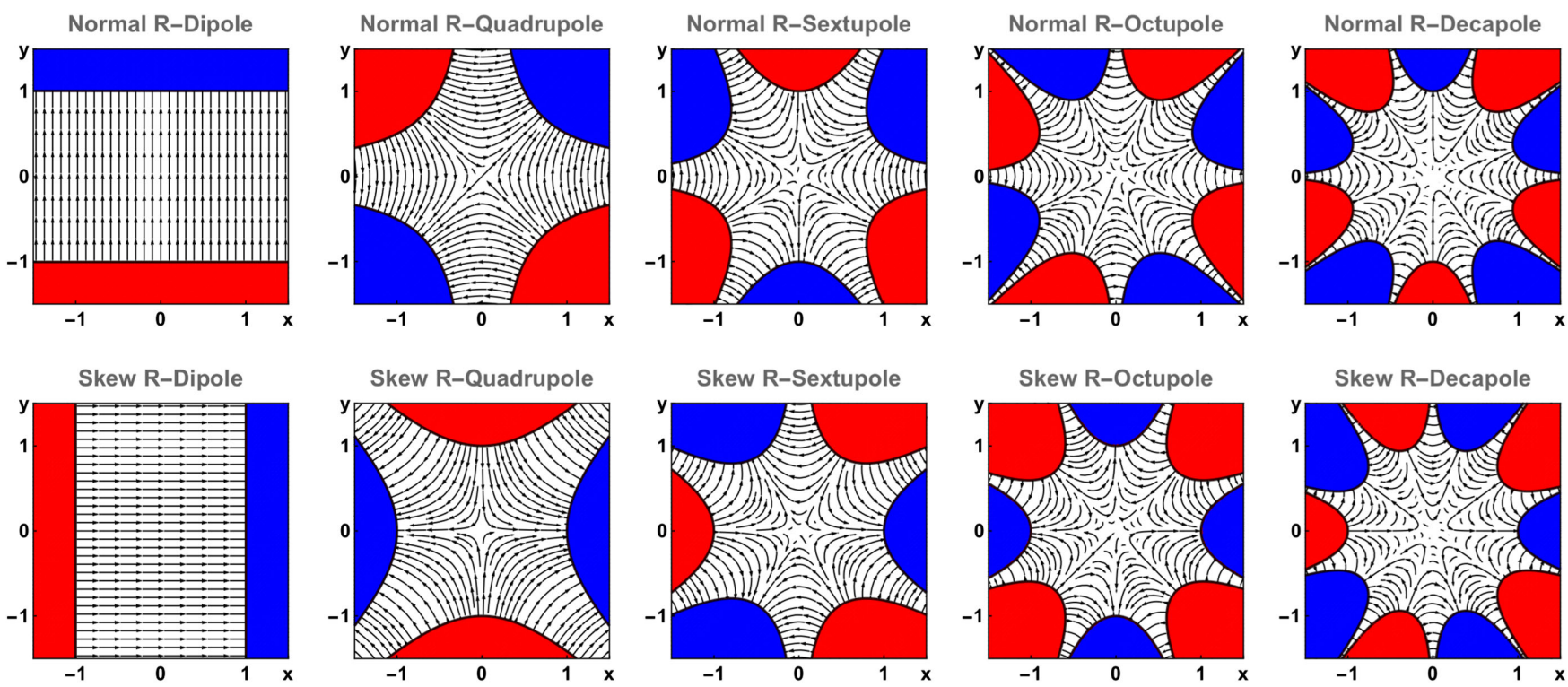

FIG. 2. Normal and skew $2 n$-pole magnets in Cartesian coordinates. Each figure shows magnetic (electric) field streamlines and poles' shape in transverse cross section. North (positive electrostatic potential) and south (negative electrostatic potential) poles are shown in red and blue and are given by $(\mathcal{B}, \mathcal{A})_{n}=\mp R_{\mathrm{p}}^{n}$ respectively, where $R_{\mathrm{p}}$ is the distance to the pole's tip. 
and underline (...) respectively. The complex scalar potentials of pure multipoles are

$$
\bar{\Omega}^{(n)}=-\bar{C}_{n} \frac{\mathcal{Z}^{n}}{n !} \quad \text { and } \quad \underline{\Omega}^{(n)}=-i \underline{C}_{n} \frac{\mathcal{Z}^{n}}{n !},
$$

where $\bar{C}_{n}$ and $\underline{C}_{n}$ are coefficients determining the strength of magnets. Corresponding vector fields are defined to have odd and even midplane symmetries:

$$
\begin{array}{lll}
\bar{F}_{\mathrm{y}}^{(n)}(x, y)=\bar{F}_{\mathrm{y}}^{(n)}(x,-y) & \text { and } & \bar{F}_{\mathrm{x}}^{(n)}(x, 0)=0, \\
\underline{F}_{\mathrm{x}}^{(n)}(x, y)=\underline{F}_{\mathrm{x}}^{(n)}(x,-y) & \text { and } & \underline{F}_{\mathrm{y}}^{(n)}(x, 0)=0 .
\end{array}
$$

Formulas for potentials and fields are listed in Table I and exact expressions are provided in Appendix E. Figure 2 shows the cross section of idealized multipole magnets.

\section{B. Multipoles in cylindrical coordinates}

In the normalized right-handed cylindrical coordinate system, when cylindrical symmetry $(\partial / \partial \theta=0)$ is imposed, the Laplace's equations reduce to

$$
\begin{aligned}
\Delta_{\curvearrowright} \Phi & =\Delta_{\perp} \Phi+\frac{1}{\rho} \frac{\partial \Phi}{\partial \rho} \\
& =\frac{\partial^{2} \Phi}{\partial \rho^{2}}+\frac{1}{\rho} \frac{\partial \Phi}{\partial \rho}+\frac{\partial^{2} \Phi}{\partial y^{2}}=0, \\
\Delta_{\curvearrowright} \mathbf{A} & =\left(\Delta_{\curvearrowright} A_{\theta}-\frac{A_{\theta}}{\rho^{2}}\right) \hat{\mathbf{e}}_{\theta} \\
& =\left(\frac{\partial^{2} A_{\theta}}{\partial \rho^{2}}+\frac{1}{\rho} \frac{\partial A_{\theta}}{\partial \rho}+\frac{\partial^{2} A_{\theta}}{\partial y^{2}}-\frac{A_{\theta}}{\rho^{2}}\right) \hat{\mathbf{e}}_{\theta}=0 .
\end{aligned}
$$

Compared to the case with Cartesian coordinates these equations look quite different from each other. In order to retain the symmetry one can note that

$$
\left(\Delta_{\curvearrowright} \mathbf{A}\right)_{\theta}=\frac{1}{\rho}\left[\frac{\partial^{2}}{\partial \rho^{2}}-\frac{1}{\rho} \frac{\partial}{\partial \rho}+\frac{\partial^{2}}{\partial y^{2}}\right]\left(\rho A_{\theta}\right)
$$

Thus looking for the solution in a form similar to harmonic homogeneous polynomials

$$
\begin{aligned}
\Phi & =-\sum_{k=0}^{n} \frac{\mathcal{F}_{n-k}(\rho)}{(n-k) !} \frac{y^{k}}{k !}\left(\bar{C}_{n} \sin \frac{k \pi}{2}+\underline{C}_{n} \cos \frac{k \pi}{2}\right), \\
A_{\theta} & =-\sum_{k=0}^{n} \frac{1}{\rho} \frac{\mathcal{G}_{n-k}(\rho)}{(n-k) !} \frac{y^{k}}{k !}\left(\bar{C}_{n} \cos \frac{k \pi}{2}-\underline{C}_{n} \sin \frac{k \pi}{2}\right),
\end{aligned}
$$

where $\mathcal{F}_{n}(\rho)$ and $\mathcal{G}_{n}(\rho)$ are the functions to be determined, one can find two recurrence equations:

$$
\begin{gathered}
\frac{\partial^{2} \mathcal{F}_{n}(\rho)}{\partial \rho^{2}}+\frac{1}{\rho} \frac{\partial \mathcal{F}_{n}(\rho)}{\partial \rho}=n(n-1) \mathcal{F}_{n-2}(\rho), \\
\frac{\partial^{2} \mathcal{G}_{n}(\rho)}{\partial \rho^{2}}-\frac{1}{\rho} \frac{\partial \mathcal{G}_{n}(\rho)}{\partial \rho}=n(n-1) \mathcal{G}_{n-2}(\rho) .
\end{gathered}
$$

$\mathcal{F}_{n}$ and $\mathcal{G}_{n}$ are related to each other through

$$
\mathcal{G}_{n-1}=\frac{1}{n} \rho \frac{\partial \mathcal{F}_{n}}{\partial \rho} \quad \text { and } \quad \mathcal{F}_{n-1}=\frac{1}{n} \frac{1}{\rho} \frac{\partial \mathcal{G}_{n}}{\partial \rho} .
$$

This allows us to construct lowering operators,

$$
\begin{gathered}
\mathcal{F}_{n}=\frac{1}{(n+1)(n+2)}\left[\frac{1}{\rho} \frac{\partial}{\partial \rho}\left(\rho \frac{\partial}{\partial \rho}\right)\right] \mathcal{F}_{n+2}, \\
\mathcal{G}_{n}=\frac{1}{(n+1)(n+2)}\left[\rho \frac{\partial}{\partial \rho}\left(\frac{1}{\rho} \frac{\partial}{\partial \rho}\right)\right] \mathcal{G}_{n+2},
\end{gathered}
$$

and corresponding raising operators

$$
\begin{aligned}
& \mathcal{F}_{n}=n(n-1) \int_{1}^{\rho} \frac{1}{\rho} \int_{1}^{\rho} \rho \mathcal{F}_{n-2} \mathrm{~d} \rho \mathrm{d} \rho, \\
& \mathcal{G}_{n}=n(n-1) \int_{1}^{\rho} \rho \int_{1}^{\rho} \frac{1}{\rho} \mathcal{G}_{n-2} \mathrm{~d} \rho \mathrm{d} \rho,
\end{aligned}
$$

where the lower limits take care of two arbitrary constants of integration. These operators can be used to recursively calculate all members of $\mathcal{F}$ - and $\mathcal{G}$-functions. An additional constraint to terminate recurrences defines lowest orders $n=0,1$ as

$$
\mathcal{F}_{0}=1, \quad \mathcal{F}_{1}=\ln \rho, \quad \mathcal{G}_{0}=1, \quad \mathcal{G}_{1}=\left(\rho^{2}-1\right) / 2 .
$$

The first ten members of $\mathcal{F}_{n}$ and $\mathcal{G}_{n}$ are listed in Tables II and III and are shown in Fig. 3; in Appendix F one can find Taylor series of these functions at $\rho=1$. The difference relation for $\mathcal{F}_{n}$ including first members has been found by Edwin M. McMillan and I would like to acknowledge his result by giving them a name of McMillan radial harmonics. In addition to his results, adjoint McMillan radial harmonics, $\mathcal{G}_{n}$, are introduced in order to provide the symmetry in the description between electric and magnetic fields.

Finally, in order to define the set of functions for pure S-multipoles (Table IV) we will define sector harmonics:

$$
\begin{aligned}
& \mathcal{A}_{n}^{(\mathrm{e})}(\rho, y)=\sum_{k=0}^{n}\left(\begin{array}{l}
n \\
k
\end{array}\right) \mathcal{F}_{n-k}(\rho) y^{k} \cos \frac{k \pi}{2}, \\
& \mathcal{A}_{n}^{(\mathrm{m})}(\rho, y)=\sum_{k=0}^{n}\left(\begin{array}{l}
n \\
k
\end{array}\right) \frac{\mathcal{G}_{n-k}(\rho)}{\rho} y^{k} \cos \frac{k \pi}{2}, \\
& \mathcal{B}_{n}^{(\mathrm{e})}(\rho, y)=\sum_{k=0}^{n}\left(\begin{array}{l}
n \\
k
\end{array}\right) \mathcal{F}_{n-k}(\rho) y^{k} \sin \frac{k \pi}{2}, \\
& \mathcal{B}_{n}^{(\mathrm{m})}(\rho, y)=\sum_{k=0}^{n}\left(\begin{array}{l}
n \\
k
\end{array}\right) \frac{\mathcal{G}_{n-k}(\rho)}{\rho} y^{k} \sin \frac{k \pi}{2},
\end{aligned}
$$

obeying the differential relations

$$
\begin{aligned}
& n(\mathcal{A}, \mathcal{B})_{n-1}^{(\mathrm{e})}= \pm \frac{\partial(\mathcal{B}, \mathcal{A})_{n}^{(\mathrm{e})}}{\partial y}=\frac{1}{\rho} \frac{\partial\left(\rho(\mathcal{A}, \mathcal{B})_{n}^{(\mathrm{m})}\right)}{\partial \rho} \\
& n(\mathcal{A}, \mathcal{B})_{n-1}^{(\mathrm{m})}= \pm \frac{1}{\varnothing} \frac{\partial\left(\phi(\mathcal{B}, \mathcal{A})_{n}^{(\mathrm{m})}\right)}{\partial y}=\frac{\partial(\mathcal{A}, \mathcal{B})_{n}^{(\mathrm{e})}}{\partial \rho} .
\end{aligned}
$$

Figure 4 shows the cross section of idealized magnetic multipoles poles and their corresponding fields. The first 
TABLE II. First ten members of $\mathcal{F}$-functions.

\begin{tabular}{lc}
\hline \hline$n$ & $\mathcal{F}_{n}(\rho)$ \\
\hline 0 & 1 \\
1 & $\ln \rho$ \\
2 & $\frac{1}{2}\left(\rho^{2}-1\right)-\ln \rho$ \\
3 & $\frac{3}{2}\left[-\left(\rho^{2}-1\right)+\left(\rho^{2}+1\right) \ln \rho\right]$ \\
4 & $3\left[\frac{1}{8}\left(\rho^{4}-1\right)+\frac{1}{2}\left(\rho^{2}-1\right)-\left(\rho^{2}+\frac{1}{2}\right) \ln \rho\right]$ \\
5 & $\frac{15}{2}\left[-\frac{3}{8}\left(\rho^{4}-1\right)+\left(\frac{1}{4} \rho^{4}+\rho^{2}+\frac{1}{4}\right) \ln \rho\right]$ \\
6 & $\frac{45}{4}\left[\frac{1}{36}\left(\rho^{6}-1\right)+\frac{1}{2}\left(\rho^{4}-1\right)-\frac{1}{4}\left(\rho^{2}-1\right)-\left(\frac{1}{2} \rho^{4}+\rho^{2}+\frac{1}{6}\right) \ln \rho\right]$ \\
7 & $\frac{315}{16}\left[-\frac{11}{54}\left(\rho^{6}-1\right)-\frac{1}{2} \rho^{2}\left(\rho^{2}-1\right)+\left\{\frac{1}{9}\left(\rho^{6}+1\right)+\rho^{2}\left(\rho^{2}+1\right)\right\} \ln \rho\right]$ \\
8 & $\frac{105}{4}\left[\frac{1}{96}\left(\rho^{8}-1\right)+\frac{4}{9}\left(\rho^{6}-1\right)+\frac{3}{8}\left(\rho^{4}-1\right)-\frac{2}{3}\left(\rho^{2}-1\right)-\left(\frac{1}{3} \rho^{6}+\frac{3}{2} \rho^{4}+\rho^{2}+\frac{1}{12}\right) \ln \rho\right]$ \\
9 & $\frac{315}{8}\left[-\frac{25}{192}\left(\rho^{8}-1\right)-\frac{5}{6} \rho^{2}\left(\rho^{4}-1\right)+\left\{\frac{1}{16}+\rho^{2}\left(\frac{\rho^{2}}{2}+1\right)\left(\frac{1}{8} \rho^{4}+\frac{7}{4} \rho^{2}+1\right)\right\} \ln \rho\right]$ \\
\hline \hline
\end{tabular}

TABLE III. First ten members of $\mathcal{G}$-functions.

\begin{tabular}{lc}
\hline \hline$n$ & $\mathcal{G}_{n}(\rho)$ \\
\hline 0 & 1 \\
1 & $\frac{1}{2}\left(\rho^{2}-1\right)$ \\
2 & $1\left[-\frac{1}{2}\left(\rho^{2}-1\right)+\rho^{2} \ln \rho\right]$ \\
3 & $\frac{3}{2}\left[\frac{1}{4}\left(\rho^{4}-1\right)-\rho^{2} \ln \rho\right]$ \\
4 & $3\left[-\frac{5}{8}\left(\rho^{4}-1\right)+\frac{1}{2}\left(\rho^{2}-1\right)+\rho^{2}\left(\frac{\rho^{2}}{2}+1\right) \ln \rho\right]$ \\
5 & $\frac{15}{4}\left[\frac{1}{12}\left(\rho^{6}-1\right)+\frac{3}{4} \rho^{2}\left(\rho^{2}-1\right)-\rho^{2}\left(\rho^{2}+1\right) \ln \rho\right]$ \\
6 & $\frac{45}{8}\left[-\frac{5}{9}\left(\rho^{6}-1\right)-\frac{1}{2}\left(\rho^{4}-1\right)+\left(\rho^{2}-1\right)+\rho^{2}\left(\frac{1}{3} \rho^{4}+2 \rho^{2}+1\right) \ln \rho\right]$ \\
7 & $\frac{105}{16}\left[\frac{1}{24}\left(\rho^{8}-1\right)+\frac{7}{6} \rho^{2}\left(\rho^{4}-1\right)-\left(\rho^{6}+3 \rho^{4}+\rho^{2}\right) \ln \rho\right]$ \\
8 & $\frac{35}{4}\left[-\frac{47}{96}\left(\rho^{8}-1\right)-2\left(\rho^{6}-1\right)+\frac{9}{8}\left(\rho^{4}-1\right)+\frac{4}{3}\left(\rho^{2}-1\right)+\rho^{2}\left(\frac{1}{4} \rho^{6}+3 \rho^{4}+\frac{9}{2} \rho^{2}+1\right) \ln \rho\right]$ \\
9 & $\frac{315}{32}\left[\frac{1}{40}\left(\rho^{10}-1\right)+\frac{35}{24} \rho^{2}\left(\rho^{6}-1\right)+\frac{5}{2} \rho^{4}\left(\rho^{2}-1\right)-\left(\rho^{8}+6 \rho^{6}+6 \rho^{4}+\rho^{2}\right) \ln \rho\right]$ \\
\hline \hline
\end{tabular}

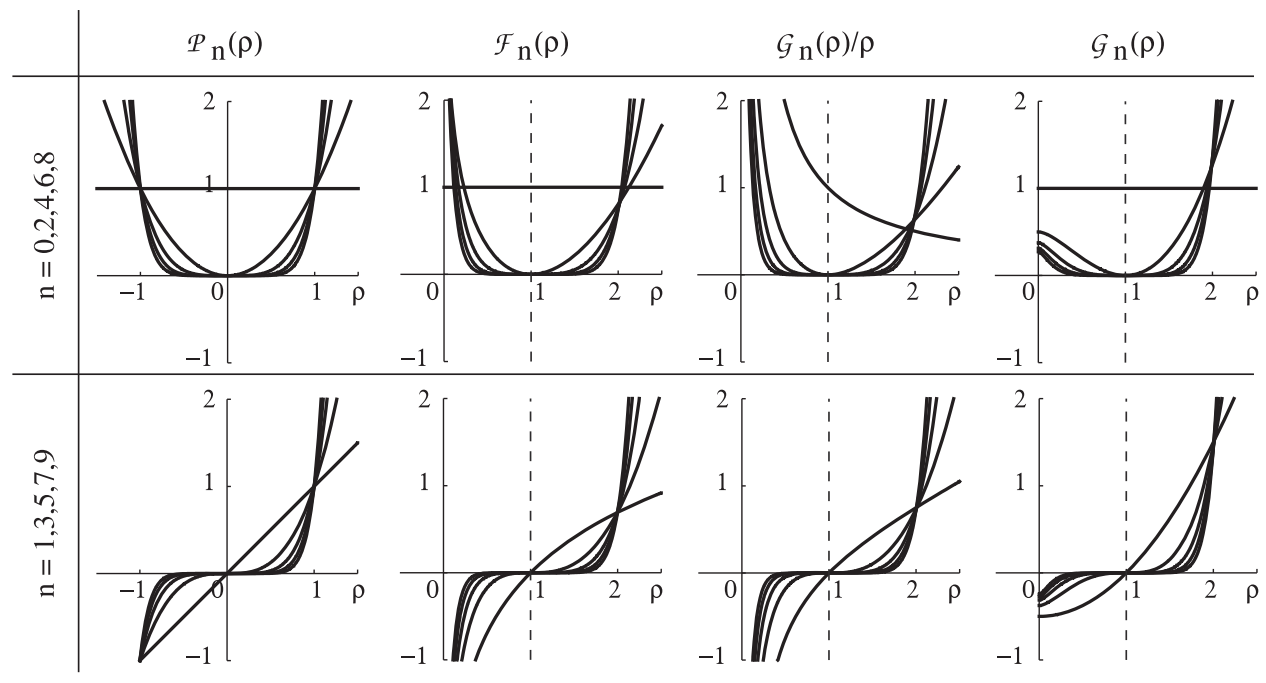

FIG. 3. First five even (top row) and odd (bottom row) members of regular polynomials $\mathcal{P}_{n}=\rho^{n}, \mathcal{F}_{n}(\rho), \frac{\mathcal{G}_{n}(\rho)}{\rho}$ and $\mathcal{G}_{n}(\rho)$ functions from the left to the right, respectively. 

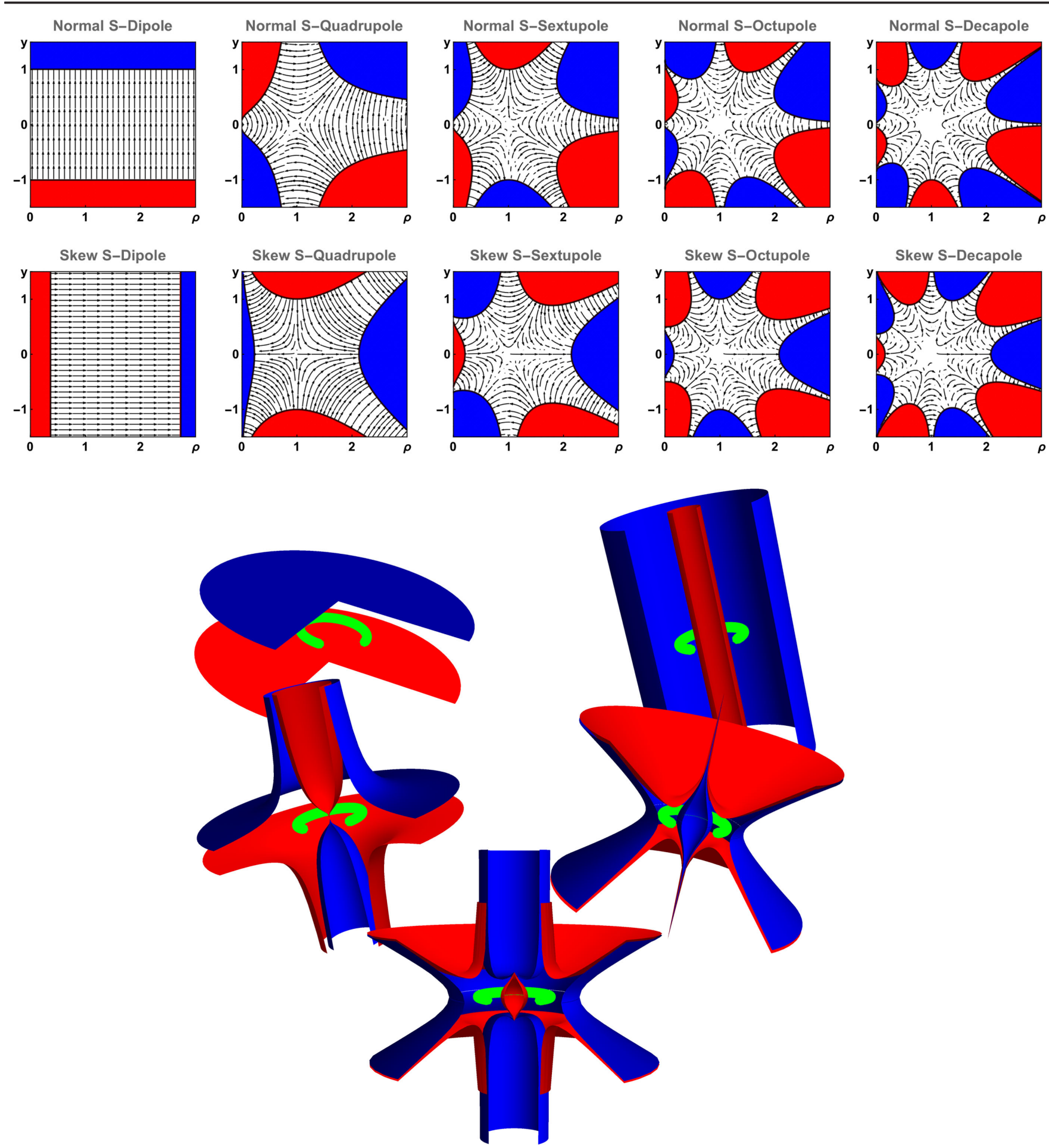

FIG. 4. Normal and skew 2n-pole magnets in cylindrical coordinates. Each figure shows magnetic (electric) field streamlines and poles' shape in the transverse cross section. North (positive electrostatic potential) and south (negative electrostatic potential) poles are shown in red and blue and given by constant levels of $(\mathcal{B}, \mathcal{A})_{n}^{(\mathrm{e})}=\mp$ const respectively, const $=1$ for this example. The bottom plot shows 3D models of sector magnets with $\theta=3 \pi / 2$ : normal and skew S-dipoles, normal and skew Squadrupoles and skew S-sextupole from the left to the right and the top to the bottom respectively. The equilibrium orbit is shown in green color. 
TABLE IV. Formulas for the scalar potential, azimuthal component of the vector potential and field components for "pure" normal and skew $2 n$-poles in cylindrical coordinates.

\begin{tabular}{lc}
\hline \hline Normal & Skew \\
\hline $\bar{\Phi}^{(n)}=-\bar{C}_{n} \frac{\mathcal{B}_{n}^{(\mathrm{e})}}{n !}$ & $\underline{\Phi}^{(n)}=-\underline{C}_{n} \frac{\mathcal{A}_{n}^{(\mathrm{e})}}{n !}$ \\
$\bar{A}_{\theta}^{(n)}=-\bar{C}_{n} \frac{\mathcal{A}_{n}^{(\mathrm{m})}}{n !}$ & $\underline{A}_{\theta}^{(n)}=\underline{C}_{n} \frac{\mathcal{B}_{n}^{(\mathrm{m})}}{n !}$ \\
$\bar{F}_{\rho}^{(n)}=\bar{C}_{n} \frac{\mathcal{B}_{n-1}^{(\mathrm{m})}}{(n-1) !}$ & $\underline{F}_{\rho}^{(n)}=\underline{C}_{n} \frac{\mathcal{A}_{n-1}^{(\mathrm{m})}}{(n-1) !}$ \\
$\bar{F}_{\mathrm{y}}^{(n)}=\bar{C}_{n} \frac{\mathcal{A}_{n-1}^{(\mathrm{e})}}{(n-1) !}$ & $\underline{F}_{\mathrm{y}}^{(n)}=-\underline{C}_{n} \frac{\mathcal{B}_{n-1}^{(\mathrm{e})}}{(n-1) !}$ \\
\hline \hline
\end{tabular}

six members of sector harmonics are listed in Table VIII of Appendix D and exact expressions for potentials and fields in Appendix E.

Finally, one can relate experimental data on the power series expansions of the fields at the reference orbit:

$$
\begin{gathered}
\left.F_{x, y}\right|_{x=0}=\left.F_{x, y}\right|_{\mathrm{eq}}+\left.\frac{y}{1 !} \frac{\partial F_{x, y}}{\partial y}\right|_{\mathrm{eq}}+\left.\frac{y^{2}}{2 !} \frac{\partial^{2} F_{x, y}}{\partial y^{2}}\right|_{\mathrm{eq}}+\cdots \\
\text { or } \\
\left.F_{x, y}\right|_{y=0}=\left.F_{x, y}\right|_{\mathrm{eq}}+\left.\frac{x}{1 !} \frac{\partial F_{x, y}}{\partial x}\right|_{\mathrm{eq}}+\left.\frac{x^{2}}{2 !} \frac{\partial^{2} F_{x, y}}{\partial x^{2}}\right|_{\mathrm{eq}}+\cdots
\end{gathered}
$$

to strength coefficients, which allows the expansion of a general S-element in terms of harmonics (see Table V).

\section{Recurrence equations in sector coordinates}

An alternative approach to finding expansions for potentials is to use a power series ansatz. In Cartesian coordinates the use of

$$
\Phi=-\sum_{m, n \geq 0}^{\infty} V_{m, n} \frac{x^{m}}{m !} \frac{y^{n}}{n !}
$$

gives the recurrence relation

$$
V_{m+2, n}+V_{m, n+2}=0 .
$$

This equation immediately defines all coefficients. It is easy to see that, up to a common factor, the solutions coincide with harmonic homogeneous polynomials $(\mathcal{A}, \mathcal{B})_{n}$.

In sector coordinates, the same substitution for $\Phi$ and the substitution

$$
A_{\theta}=-\sum_{m, n \geq 0}^{\infty} \frac{1}{1+x} V_{m, n} \frac{x^{m}}{m !} \frac{y^{n}}{n !}
$$

\begin{tabular}{|c|c|c|c|}
\hline & $n$ & $x=0$ & $y=0$ \\
\hline \multirow[t]{9}{*}{$\bar{C}_{n}$} & 1 & $F_{y}$ & $F_{y}$ \\
\hline & 2 & $\partial_{y} F_{x}$ & $\partial_{x} F_{y}$ \\
\hline & 3 & $-\partial_{y}^{2} F_{y}$ & $\partial_{x}^{2} F_{y}+\partial_{x} F_{y}$ \\
\hline & 4 & $-\partial_{y}^{3} F_{x}$ & $\partial_{x}^{3} F_{y}+\partial_{x}^{2} F_{y}-\partial_{x} F_{y}$ \\
\hline & 5 & $\partial_{y}^{4} F_{y}$ & $\partial_{x}^{4} F_{y}+2 \partial_{x}^{3} F_{y}-\partial_{x}^{2} F_{y}+\partial_{x} F_{y}$ \\
\hline & 6 & $\partial_{y}^{5} F_{x}$ & $\partial_{x}^{5} F_{y}+2 \partial_{x}^{4} F_{y}-3 \partial_{x}^{3} F_{y}+3 \partial_{x}^{2} F_{y}-3 \partial_{x} F_{y}$ \\
\hline & 7 & $-\partial_{y}^{6} F_{y}$ & $\partial_{x}^{6} F_{y}+3 \partial_{x}^{5} F_{y}-3 \partial_{x}^{4} F_{y}+6 \partial_{x}^{3} F_{y}-9 \partial_{x}^{2} F_{y}+9 \partial_{x} F_{y}$ \\
\hline & 8 & $-\partial_{y}^{7} F_{x}$ & $\partial_{x}^{7} F_{y}+3 \partial_{x}^{6} F_{y}-6 \partial_{x}^{5} F_{y}+12 \partial_{x}^{4} F_{y}-27 \partial_{x}^{3} F_{y}+45 \partial_{x}^{2} F_{y}-45 \partial_{x} F_{y}$ \\
\hline & 9 & $\partial_{y}^{8} F_{y}$ & $\partial_{x}^{8} F_{y}+4 \partial_{x}^{7} F_{y}-6 \partial_{x}^{6} F_{y}+18 \partial_{x}^{5} F_{y}-51 \partial_{x}^{4} F_{y}+126 \partial_{x}^{3} F_{y}-225 \partial_{x}^{2} F_{y}+225 \partial_{x} F_{y}$ \\
\hline \multirow[t]{9}{*}{$\underline{C}_{n}$} & 1 & $F_{x}$ & $F_{x}$ \\
\hline & 2 & $-\partial_{y} F_{y}$ & $\partial_{x} F_{x}+F_{x}$ \\
\hline & 3 & $-\partial_{y}^{2} F_{x}$ & $\partial_{x}^{2} F_{x}+\partial_{x} F_{x}-F_{x}$ \\
\hline & 4 & $\partial_{y}^{3} F_{y}$ & $\partial_{x}^{3} F_{x}+2 \partial_{x}^{2} F_{x}-\partial_{x} F_{x}+F_{x}$ \\
\hline & 5 & $\partial_{y}^{4} F_{x}$ & $\partial_{x}^{4} F_{x}+2 \partial_{x}^{3} F_{x}-3 \partial_{x}^{2} F_{x}+3 \partial_{x} F_{x}-3 F_{x}$ \\
\hline & 6 & $-\partial_{y}^{5} F_{y}$ & $\partial_{x}^{5} F_{x}+3 \partial_{x}^{4} F_{x}-3 \partial_{x}^{3} F_{x}+6 \partial_{x}^{2} F_{x}-9 \partial_{x} F_{x}+9 F_{x}$ \\
\hline & 7 & $-\partial_{y}^{6} F_{x}$ & $\partial_{x}^{6} F_{x}+3 \partial_{x}^{5} F_{x}-6 \partial_{x}^{4} F_{x}+12 \partial_{x}^{3} F_{x}-27 \partial_{x}^{2} F_{x}+45 \partial_{x} F_{x}-45 F_{x}$ \\
\hline & 8 & $\partial_{y}^{7} F_{y}$ & $\partial_{x}^{7} F_{x}+4 \partial_{x}^{6} F_{x}-6 \partial_{x}^{5} F_{x}+18 \partial_{x}^{4} F_{x}-51 \partial_{x}^{3} F_{x}+126 \partial_{x}^{2} F_{x}-225 \partial_{x} F_{x}+225 F_{x}$ \\
\hline & 9 & $\partial_{y}^{8} F_{x}$ & $\partial_{x}^{8} F_{x}+4 \partial_{x}^{7} F_{x}-10 \partial_{x}^{6} F_{x}+30 \partial_{x}^{5} F_{x}-105 \partial_{x}^{4} F_{x}+330 \partial_{x}^{3} F_{x}-855 \partial_{x}^{2} F_{x}+1575 \partial_{x} F_{x}-1575 F_{x}$ \\
\hline
\end{tabular}

for the longitudinal component of the vector potential gives two new recurrences, respectively:

TABLE V. Relationship between coefficients determining the strength of pure normal and skew S-multipoles and power series expansion of field in radial and vertical planes on equilibrium orbit. 


$$
V_{m+2, n}+V_{m, n+2}=-(m \pm 1) V_{m+1 . n}-m V_{m-1, n+2} .
$$

The detailed approach on how to treat these equations can be found for example in [3]. In order to solve these recurrences, one can look for a solution where each term can be expressed in the form

$$
V_{i, j}=V_{i, j}^{*}+V_{i, j}^{(i+j-1)}+V_{i, j}^{(i+j-2)}+V_{i, j}^{(i+j-3)}+\cdots,
$$

where starred variables are the "design" terms given by pure multipole fields and thus satisfying

$$
V_{m+2, n}^{*}+V_{m, n+2}^{*} \equiv 0 .
$$

Other coefficients $V_{i, j}^{(k)}$ are terms induced by lower $k$ th order pure multipoles due to recurrence. Thus, in order to find an expression for a particular $2 n$-pole we will start the recurrence from the $n$th order assuming that

$$
V_{n, 0}=-V_{n-2,2}=\cdots \quad \text { or } \quad V_{n-1,1}=-V_{n-3,3}=\cdots
$$

for normal and skew elements. Then we will start exploiting the recurrence where all terms in the form $V_{i, j}^{(n)}$ for $i+j>n$ are subject to be determined.

This approach has two major disadvantages. First, in order to use the result, one will have to truncate a recurrence. As a result the potentials representing magnets no longer satisfy Laplace's equation. This violates the "physics" and should be avoided. While potentials can be approximated with any precision by keeping an appropriate number of terms, there is another issue. When solving the recurrence, at each new order one will find that an arbitrary constant $\alpha_{i} \in(0 ; 1)$ should be introduced since the system is undetermined. An additional assumption, $\left.\left(A_{s}, \Phi\right)\right|_{x=0} \propto y^{n}$, allows us to terminate or sum the series. The resulting solutions would then coincide with the ones obtained above.

\section{SUMMARY}

The scalar and vector Laplace's equations for static transverse electromagnetic fields in curvilinear orthogonal coordinates with zero and constant curvature are solved. In Cartesian coordinates these solutions are wellknown harmonic polynomials in two variables. The set of solutions in cylindrical coordinates, named sector harmonics, should not be confused with cylindrical harmonics where $\rho$-dependent terms are given by Bessel functions (which occasionally are also called cylindrical harmonics). In contrast, the radial part is given by a set of harmonics, independently introduced by Edwin M. McMillan in a "forgotten" article, and adjoint radial harmonics described in this work (in addition it was rediscovered by Mane in the beginning of the 1990s). When expanded around a circular design orbit, the sector harmonics resemble the solutions in Cartesian geometry. This set of functions has two major advantages over the traditional approach, widely used in the accelerator community, of using recurrences based on a power series ansatz. They do not require truncation and satisfy Laplace's equation exactly, and they provide a well-defined full basis of functions which can be related to any field by expansion in radial or vertical planes, see Table V. Including the model Hamiltonians for $t$ - and $s$ representations, where no assumptions but the field symmetry has been used, one can construct a numerical scheme to integrate the equations of motion. Thus, I would like to suggest the set of sector harmonics as a new basis for the description and design of sector magnets with translational symmetry along the azimuthal coordinate.

\section{ACKNOWLEDGMENTS}

The author would like to thank Leo Michelotti, Eric Stern and James Amundson for their discussions and valuable input, Alexey Burov for encouraging me to find a full family of solutions, Valeri Lebedev whose solution for electrostatic quadrupole led me to a generalization, just as was the case with Edwin M. McMillan and F. Krienen, and Sergei Nagaitsev who introduced me to McMillan's article, which helped me with the symmetric description of electromagnetic fields. FermiLab is operated by Fermi Research Alliance, LLC under Contract No. DeAC02-07CH11359 with the U.S. Department of Energy.

\section{APPENDIX A: GENERAL EQUATIONS OF MOTION IN GLOBAL LAB FRAME}

The Lagrangian of a relativistic particle of mass $m$ with an electric charge $e$ in most general static electromagnetic field is given by

$$
\mathcal{L}[\mathbf{R}, \dot{\mathbf{R}} ; t]=-\frac{m c^{2}}{\gamma(\mathbf{V})}-e \Phi(\mathbf{R})+e(\mathbf{V} \cdot \mathbf{A}(\mathbf{R})),
$$

where $\mathbf{R}=\left(Q_{1}, Q_{2}, Q_{3}\right)$ is a position vector in the configuration space of coordinates spanned by a right-handed Cartesian coordinate system, $\left\{\hat{\mathbf{E}}_{1}, \hat{\mathbf{E}}_{2}, \hat{\mathbf{E}}_{3}\right\}, \mathbf{V}=d \mathbf{R} / d t \equiv$ $\dot{\mathbf{R}}$ is a vector of matching generalized velocities, $\Phi(\mathbf{R})$ and $\mathbf{A}(\mathbf{R})$ are the electric scalar and magnetic vector potentials respectively, and

$$
\gamma(\mathbf{V})=\frac{1}{\sqrt{1-\beta(\mathbf{V})^{2}}}
$$

is the relativistic Lorentz factor, with $\beta(\mathbf{V})=|\mathbf{V}| / c$.

Substituting the Lagrangian into the Euler-Lagrange equations (Lagrange's equations of the second kind) 


$$
\frac{\mathrm{d}}{\mathrm{d} t} \frac{\partial \mathcal{L}}{\partial \dot{\mathbf{R}}}-\frac{\partial \mathcal{L}}{\partial \mathbf{R}}=0
$$

with shorthand notation

$$
\frac{\partial}{\partial \mathbf{a}}=\left(\frac{\partial}{\partial a_{1}}, \frac{\partial}{\partial a_{2}}, \frac{\partial}{\partial a_{3}}\right)
$$

representing a vector of partial derivatives with respect to the indicated variables, gives the equation of motion which is the relativistic form of the Lorentz force

$$
\mathbf{F}=e[\mathbf{E}+(\mathbf{V} \times \mathbf{B})]
$$

or explicitly

$$
\frac{\mathrm{d}}{\mathrm{d} t}\left(\gamma m \dot{Q}_{i}\right)=e\left(E_{i}+\epsilon_{i j k} \dot{Q}_{j} B_{k}\right)
$$

Electric and magnetic fields are related to the scalar electric and vector magnetic potentials through the usual gradient and curl operators:

$$
\begin{aligned}
& \mathbf{E}=\left(E_{1}, E_{2}, E_{3}\right) \equiv-\nabla \Phi, \\
& \mathbf{B}=\left(B_{1}, B_{2}, B_{3}\right) \equiv \nabla \times \mathbf{A} .
\end{aligned}
$$

The corresponding Hamiltonian formulation employs phase space coordinates $(\mathbf{P}, \mathbf{Q})$, where $\mathbf{P}$ is the particle's canonical (total) momentum,

$$
\mathbf{P} \equiv \frac{\partial \mathcal{L}}{\partial \dot{\mathbf{R}}}=\boldsymbol{\Pi}+e \mathbf{A}
$$

with $\Pi=\gamma m \mathbf{V}$ being the particle's kinematic momentum. The Hamiltonian might be constructed using the Legendre transformation of $\mathcal{L}$ :

$$
\begin{aligned}
\mathcal{H}[\mathbf{P}, \mathbf{Q} ; t] & =\mathbf{V} \cdot \mathbf{P}-\mathcal{L}=\sum_{i=1}^{3} \dot{Q}_{i} P_{i}-\mathcal{L} \\
& =c \sqrt{m^{2} c^{2}+(\mathbf{P}-e \mathbf{A})^{2}}+e \Phi .
\end{aligned}
$$

The time evolution of the system is given by Hamilton's equations

$$
\frac{\mathrm{d} \mathbf{P}}{\mathrm{d} t}=-\frac{\partial \mathcal{H}}{\partial \mathbf{Q}} \quad \text { and } \quad \frac{\mathrm{d} \mathbf{Q}}{\mathrm{d} t}=\frac{\partial \mathcal{H}}{\partial \mathbf{P}}
$$

or equivalently

$$
\begin{aligned}
\dot{\mathbf{Q}} & =c \frac{\mathbf{P}-e \mathbf{A}}{\sqrt{m^{2} c^{2}+(\mathbf{P}-e \mathbf{A})^{2}}}, \\
\dot{\mathbf{P}} & =e(\nabla \mathbf{A}) \cdot \dot{\mathbf{Q}}-e \nabla \Phi .
\end{aligned}
$$

\section{APPENDIX B: GLOBAL COORDINATES ASSOCIATED WITH FRENET-SERRET FRAME}

The model of accelerator assumes the specification of a reference orbit designed for a particle with certain equilibrium energy and assignment of beam line elements placed along it. In the case of a circular accelerator the closed orbit of a machine with alignment errors in general will not coincide with reference orbit. For most accelerator needs (except e.g. helical orbits for muon cooling) the designed orbit is piecewise flat function, which means that it consists of a series of curves with zero torsion; moreover, usually, these curves are straight lines and circular arcs. In order to better exploit the geometry of beam motion and symmetry of electromagnetic fields we will introduce the local Frenet-Serret frame attached to the equilibrium orbit and new global coordinates associated with it (see Fig. 5).

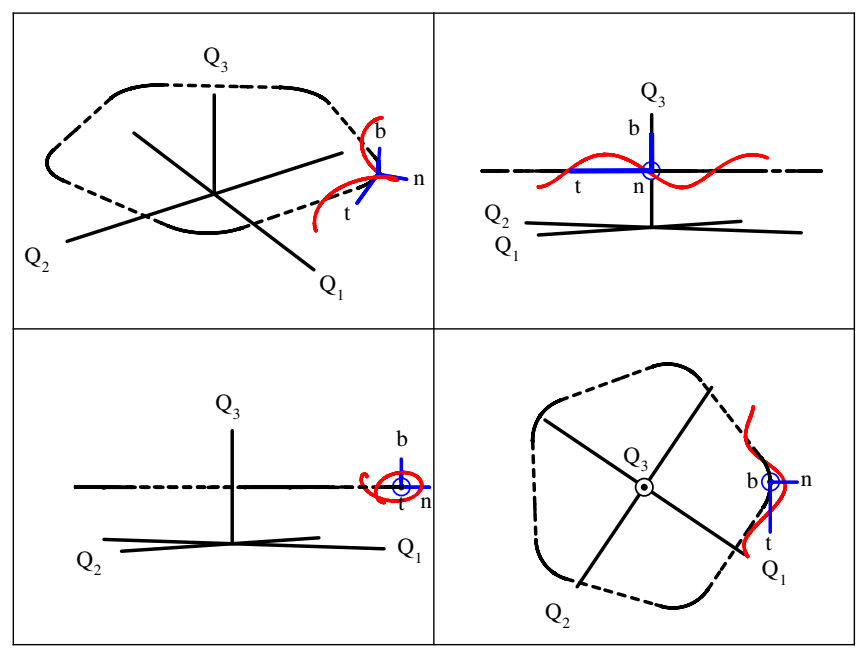

FIG. 5. Schematic plot of a reference orbit for an accelerator consisting of five straight and five $72^{\circ}$ curved sections. Lab frame and local Frenet-Serret frames are shown in black and blue colors respectively. The test particle winding the equilibrium orbit is shown in red.

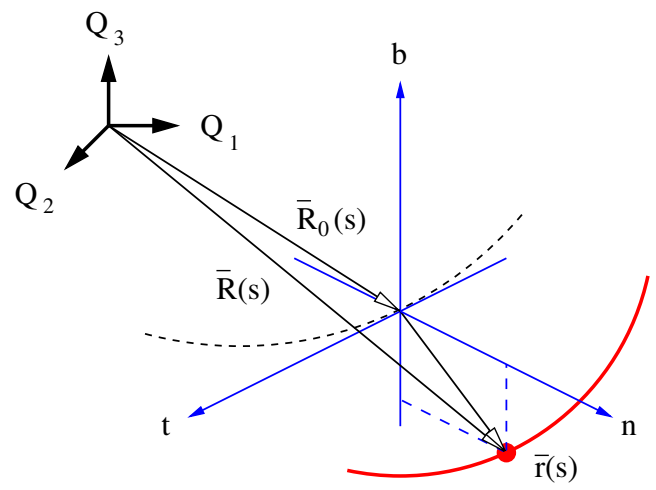

FIG. 6. Illustration of a test particle's position vector expressed as a transverse, i.e. for fixed $q_{3}$, displacement from equilibrium orbit. 
We identify a particular curve segment in space as the local "reference path" (See Fig. 6). A particle whose orbit would follow that curve perfectly is a "reference particle" and its orbit, the "reference orbit." Let $\mathbf{R}_{0}(t)$ be its position vector as a function of time. Arclength along the path can be expressed as

$$
s(t)=\int_{0}^{t}\left|\dot{\mathbf{R}}_{0}(t)\right| \mathrm{d} t
$$

and $\mathbf{R}_{0}$ can be parametrized as a function of $s$ rather than $t$. The local Frenet-Serret coordinate frame with origin at $\mathbf{R}_{0}$ has right-handed orthonormal basis vectors, $\{\hat{\mathbf{n}}, \hat{\mathbf{b}}, \hat{\mathbf{t}}\}$ (or TNB frame), defined as follows: (i) tangent unit vector

$$
\hat{\mathbf{t}}=\frac{\mathrm{d} \mathbf{R}_{0}(s)}{\mathrm{d} s},
$$

(ii) outward-pointing normal unit vector

$$
\hat{\mathbf{n}}=-\frac{1}{\kappa(s)} \frac{\mathrm{d} \hat{\mathbf{t}}}{\mathrm{d} s},
$$

(iii) and binormal unit vector

$$
\hat{\mathbf{b}}=\hat{\mathbf{t}} \times \hat{\mathbf{n}},
$$

where $\kappa=|\mathrm{d} \hat{\mathbf{t}} / \mathrm{d} s|$ defines the local curvature of the equilibrium orbit. Using the Frenet-Serret formulas describing the derivatives of unit vectors in terms of each other,

$$
\mathrm{d}\left[\begin{array}{c}
\hat{\mathbf{t}} \\
\hat{\mathbf{n}} \\
\hat{\mathbf{b}}
\end{array}\right]=\left[\begin{array}{ccc}
0 & -\kappa & 0 \\
\kappa & 0 & \tau \\
0 & -\tau & 0
\end{array}\right]\left[\begin{array}{c}
\hat{\mathbf{t}} \\
\hat{\mathbf{n}} \\
\hat{\mathbf{b}}
\end{array}\right] \mathrm{d} s,
$$

where $\tau(s)$ is the torsion of an equilibrium orbit which measures the failure of a curve to be planar, the position vector of a test particle and its infinitesimal can be expressed as a displacement from an equilibrium orbit,

$$
\begin{aligned}
\mathbf{R} & =\mathbf{R}_{0}(s)+\mathbf{r}(s)=\mathbf{R}_{0}(s)+q_{1} \hat{\mathbf{n}}+q_{2} \hat{\mathbf{b}} \\
\mathrm{d} \mathbf{R} & =\hat{\mathbf{n}} \mathrm{d} q_{1}+\hat{\mathbf{b}} \mathrm{d} q_{2}+\left(1+\kappa q_{1}\right) \hat{\mathbf{t}} \mathrm{d} q_{3}+\tau\left(q_{1} \hat{\mathbf{b}}-q_{2} \hat{\mathbf{n}}\right) \mathrm{d} q_{3},
\end{aligned}
$$

where $\left(q_{1}, q_{2}, q_{3}\right)$ are local curvilinear coordinates spanned on $(\hat{\mathbf{n}}, \hat{\mathbf{b}}, \hat{\mathbf{t}})$. One can see that in the case of flat orbit, i.e. $\tau=0$, the local Frenet-Serret frame can be associated with global orthogonal coordinate system with a line element in a form

$$
\mathrm{d} \mathbf{l}=h_{1} \hat{\mathbf{e}}_{1} \mathrm{~d} q_{1}+h_{1} \hat{\mathbf{e}}_{1} \mathrm{~d} q_{1}+h_{1} \hat{\mathbf{e}}_{1} \mathrm{~d} q_{1},
$$

where scale factors are $h_{1}=h_{2}=1$ and $h \equiv h_{3}=1+\kappa q_{1}$.

The use of global coordinates with the metric provided by the local Frenet-Serret frame allows one to write the Lagrangian as

$$
\mathcal{L}[\mathbf{r}, \dot{\mathbf{r}} ; t]=-m c^{2} \sqrt{1-\frac{v^{2}}{c^{2}}}-e \Phi+e \mathbf{v} \cdot \mathbf{A},
$$

where $\mathbf{v}=\left(\dot{q}_{1}, \dot{q}_{2}, h \dot{q}_{3}\right)$ is the particle's velocity expressed in new coordinates. Thus the equation of motion is

$$
\frac{\mathrm{d}}{\mathrm{d} t}(\gamma m \mathbf{v})=e\left(\mathbf{E}+\epsilon_{i j k} \hat{\mathbf{e}}_{i} v_{j} B_{k}\right)+\gamma m \dot{q}_{3}^{2} \mathbf{K},
$$

where the vector in the right-hand side of the equation is defined as

$$
\mathbf{K}=\left(\kappa h, 0, \kappa^{\prime} q_{1}\right),
$$

and $\kappa^{\prime} \equiv d \kappa / d q_{3}$ is the derivative of $\kappa$ with respect to the longitudinal coordinate. Derivatives of potentials using expressions for differential operators in curvilinear orthogonal coordinates form Table VI. Calculating components of the new canonical momenta

$$
\frac{p_{i}}{h_{i}} \equiv \frac{1}{h_{i}} \frac{\partial \mathcal{L}}{\partial \dot{q}_{i}}=\gamma m v_{i}+e \mathbf{A}_{i}(\mathbf{r})
$$

allows one to rewrite the Hamiltonian

$$
\mathcal{H}[\mathbf{p}, \mathbf{q} ; t]=c \sqrt{m^{2} c^{2}+\sum_{i=1}^{3}\left(\frac{p_{i}-e h_{i} A_{i}}{h_{i}}\right)^{2}}+e \Phi
$$

and equations of motion

$$
\begin{aligned}
\dot{q}_{i} \times h_{i} & =\frac{c^{2}}{\mathcal{H}-e \Phi} \frac{p_{i}-e h_{i} A_{i}}{h_{i}} \\
\dot{p}_{i} / h_{i} & =\frac{c^{2}}{\mathcal{H}-e \Phi}\left[e \epsilon_{i j k} \frac{p_{j}}{h_{j}} B_{k}+\frac{K_{i}}{h^{2}}\left(\frac{p_{3}-e h A_{3}}{h}\right)^{2}\right]+e E_{i} .
\end{aligned}
$$

Further simplifications can be made by specifying a type of the field or a certain symmetry. We will restrict ourself to the case of transverse electromagnetic fields. In orthogonal curvilinear coordinate system associated with Serret-Frenet frame these are the fields with translation symmetry along longitudinal coordinate $q_{3}$. Thus, the scalar and vector potentials are a function of transverse coordinates only and we shall assume the vector potential has only one nonvanishing component which is $A_{3}$. Taken together, these imply the gauge condition, $\nabla \cdot \mathbf{A}=0$. Both scalar and vector potentials satisfy Laplace's equation,

$$
\begin{aligned}
& \Delta \Phi=\frac{1}{h}\left[\frac{\partial}{\partial q_{1}}\left(h \frac{\partial \Phi}{\partial q_{1}}\right)+\frac{\partial}{\partial q_{2}}\left(h \frac{\partial \Phi}{\partial q_{2}}\right)\right]=0, \\
& \Delta \mathbf{A}=\frac{\partial}{\partial q_{1}}\left[\frac{1}{h} \frac{\partial\left(h A_{3}\right)}{\partial q_{1}}\right]+\frac{\partial}{\partial q_{2}}\left[\frac{1}{h} \frac{\partial\left(h A_{3}\right)}{\partial q_{2}}\right]=0 .
\end{aligned}
$$


The corresponding fields are given by Maxwell equations

$$
\begin{aligned}
\mathbf{E} & =-\nabla \Phi, & \mathbf{B} & =\nabla \times \mathbf{A}, r i \\
E_{1} & =-\frac{\partial \Phi}{\partial q_{1}}, & B_{1} & =\frac{1}{h} \frac{\partial\left(h A_{3}\right)}{\partial q_{2}}, \\
E_{2} & =-\frac{\partial \Phi}{\partial q_{2}}, & B_{2} & =-\frac{1}{h} \frac{\partial\left(h A_{3}\right)}{\partial q_{1}},
\end{aligned}
$$

with differential operators defined for the orthogonal curvilinear coordinate system (see Table VI in Appendix C).

In the case of pure electric or magnetic fields further simplifications can be applied. For numerical integration purposes, it is convenient to have a Hamiltonian in the form of a sum of "kinetic" and "potential" energies, so that potentials will be separated from momentum coordinates. In this case, one can easily construct a symplectic integrator consisting of "drifts" and "kicks" associated with kinetic and potential terms respectively (e.g. [7]). Depending on the field type two models can be employed.

\section{1. $t$-representation}

For a pure electric field, when the curvature is constant $(d \kappa / d s=0)$, not only the Hamiltonian but also $p_{3}$ is an invariant of motion, and the problem is essentially two dimensional. Measuring time in units of $c t$ and normalizing the transverse momenta by the longitudinal component, $\tilde{p}_{1,2}=p_{1,2} / p_{3}$, one has

$\mathrm{H}[\tilde{\mathbf{p}}, \mathbf{q} ; c t]=\frac{1}{h} \sqrt{\frac{p_{3}^{2}+h^{2} m^{2} c^{2}}{p_{3}^{2}}+h^{2}\left(\tilde{p}_{1}^{2}+\tilde{p}_{2}^{2}\right)}+\frac{e}{p_{3} c} \Phi$.

We will call this model Hamiltonian the $t$-representation; with no assumptions made, but the field symmetry, we derived general equations of motion which can be used for the basis for the construction of the symplectic integrator. In a paraxial approximation, $\tilde{p}_{1,2} \ll 1$, and for $p_{1,2} \gg m c$ the form is significantly simpler, and a limit of straight coordinates when $h=1$ is obvious:

$$
\mathrm{H}[\tilde{\mathbf{p}}, \mathbf{q} ; c t] \approx h\left(\frac{\tilde{p}_{1}^{2}}{2}+\frac{\tilde{p}_{2}^{2}}{2}\right)+\frac{1}{h}+\frac{e}{p_{3} c} \Phi
$$

\section{2. $s$-representation}

For a pure magnetic field the Hamiltonian is harder to exploit since it has only a square root and thus no terms to split. Introducing an extended Hamiltonian with a new fictitious orbit ("time") parameter, $\tau$, where the old independent variable and old Hamiltonian with a negative sign will be treated as an additional pair of canonically conjugated coordinates, $(-\mathcal{H}, t)$, one has

$$
\begin{aligned}
0 & \equiv \mathcal{O}\left[p_{1}, p_{2}, p_{3},-\mathcal{H} ; q_{1}, q_{2}, q_{3}, t ; \tau\right] \\
& =c \sqrt{m^{2} c^{2}+p_{1}^{2}+p_{2}^{2}+\left(\frac{p_{3}-e h A_{3}}{h}\right)^{2}}-\mathcal{H} .
\end{aligned}
$$

Integration of the additional equations of motion gives

$$
\mathcal{H} \text { is invariant, and } t=\tau+C_{0} .
$$

We can set the arbitrary constant of integration, $C_{0}=0$.

Continuing to assume that the curvature is constant, as was done in the case of an electric field, the longitudinal component of momentum is conserved. We shall use $-p_{3}$ as a new Hamiltonian, reducing the number of degrees of freedom back to 3 by using $q_{3}$ as a new independent variable:

$$
\begin{aligned}
-p_{3} & \equiv \mathcal{K}\left[p_{1}, p_{2},-\mathcal{H} ; q_{1}, q_{2}, t ; q_{3}\right] \\
& =-h \sqrt{\left(\frac{\mathcal{H}}{c}\right)^{2}-m^{2} c^{2}-p_{1}^{2}-p_{2}^{2}}-e h A_{3} .
\end{aligned}
$$

The use of generating function

$$
G_{2}(t,-\Pi)=-t \sqrt{\Pi^{2} c^{2}+\left(m c^{2}\right)^{2}}
$$

will allow one to use the full kinetic momentum $-\Pi$ of a particle instead of $-\mathcal{H}$ as one of canonical momentums:

$$
\mathcal{K}\left[p_{1}, p_{2},-\Pi ; q_{1}, q_{2}, l ; q_{3}\right]=-h \sqrt{\Pi^{2}-p_{1}^{2}-p_{2}^{2}}-e h A_{3},
$$

where corresponding canonical coordinate is a particle's traversed path $l=-\partial G_{2} / \partial \Pi=\beta c t$.

Since the Hamiltonian does not explicitly depend on $l$, the full momentum $\Pi$ is conserved and we can exclude associated degrees of freedom using the further renormalization of the Hamiltonian $\mathcal{K} \rightarrow \mathrm{K} \equiv \mathcal{K} / \Pi$, which can be achieved by renormalizing transverse components of canonical momentums $p_{1,2} \rightarrow \tilde{p}_{1,2}=p_{1,2} / \Pi$ :

$$
\begin{aligned}
-\frac{p_{3}}{\Pi} & \equiv \mathrm{K}\left[\tilde{p}_{1}, \tilde{p}_{2} ; q_{1}, q_{2} ; q_{3}\right] \\
& =-h \sqrt{1-\tilde{p}_{1}^{2}-\tilde{p}_{2}^{2}}-\frac{e}{\Pi} h A_{3} .
\end{aligned}
$$

We will call this model Hamiltonian s-representation since the longitudinal coordinate (sometimes referred to as the natural parameter along equilibrium orbit, s) is used as a time parameter. This representation is convenient to use for the numerical integrator construction for transverse magnetic fields. The paraxial approximation, $\tilde{p}_{1,2} \ll 1$, gives

$$
\mathrm{K}\left[\tilde{\mathbf{p}}, \mathbf{q} ; q_{3}\right] \approx h\left(\frac{\tilde{p}_{1}^{2}}{2}+\frac{\tilde{p}_{2}^{2}}{2}\right)-h-\frac{e}{\Pi} h A_{3} .
$$




\section{APPENDIX C: DIFFERENTIAL OPERATORS IN ORTHOGONAL COORDINATES}

Differential operators in orthogonal curvilinear coordinates are listed in Table VI.

TABLE VI. Differential operators in general orthogonal coordinates $\left(q_{1}, q_{2}, q_{3}\right)$ where $H=h_{1} h_{2} h_{3}$, and its expressions in orthogonal coordinates associated with Serret-Frenet frame.

\begin{tabular}{lll}
\hline \hline Operation & Notation & \multicolumn{1}{c}{ Expression } \\
\hline Gradient & $\nabla \phi$ & $\sum_{k=1}^{3} \frac{1}{h_{k}} \frac{\partial \phi}{\partial q^{k}} \hat{\mathbf{e}}_{k}$ \\
Divergence & $\frac{\partial \phi}{\partial q_{1}} \hat{\mathbf{e}}_{1}+\frac{\partial \phi}{\partial q_{2}} \hat{\mathbf{e}}_{2}+\frac{1}{h} \frac{\partial \phi}{\partial q_{3}} \hat{\mathbf{e}}_{3}$ \\
& $\nabla \cdot \mathbf{F}$ & $\sum_{k=1}^{3} \frac{1}{H} \frac{\partial}{\partial q^{k}}\left(\frac{H}{h_{k}} F_{k}\right)$ \\
Curl & $\frac{1}{h}\left[\frac{\partial\left(h F_{1}\right)}{\partial q_{1}}+\frac{\partial\left(h F_{2}\right)}{\partial q_{2}}+\frac{\partial F_{3}}{\partial q_{3}}\right]$ \\
& $\sum_{k=1}^{3} \frac{h_{k} \hat{\mathbf{e}}_{k}}{H} \epsilon_{i j k} \frac{\partial}{\partial q^{i}}\left(h_{j} F_{j}\right)$ \\
Scalar Laplacian & & $\frac{1}{h}\left[\frac{\partial\left(h F_{3}\right)}{\partial q_{2}}-\frac{\partial F_{2}}{\partial q_{3}}\right] \hat{\mathbf{e}}_{1}+\frac{1}{h}\left[\frac{\partial F_{1}}{\partial q_{3}}-\frac{\partial\left(h F_{3}\right)}{\partial q_{1}}\right] \hat{\mathbf{e}}_{2}+\left[\frac{\partial F_{2}}{\partial q_{1}}-\frac{\partial F_{1}}{\partial q_{2}} \hat{\mathbf{e}}_{3}\right.$ \\
& $\Delta \phi=\nabla \cdot(\nabla \phi)$ & $\sum_{k=1}^{3} \frac{1}{H} \frac{\partial}{\partial q^{k}}\left(\frac{H}{h_{k}^{2}} \frac{\partial \phi}{\partial q^{k}}\right)$ \\
Vector Laplacian & $\frac{1}{h}\left[\frac{\partial}{\partial q_{1}}\left(h \frac{\partial \phi}{\partial q_{1}}\right)+\frac{\partial}{\partial q_{2}}\left(h \frac{\partial \phi}{\partial q_{2}}\right)+\frac{\partial}{\partial q_{3}}\left(\frac{1}{h} \frac{\partial \phi}{\partial q_{3}}\right)\right]$ \\
\hline \hline
\end{tabular}

\section{APPENDIX D: HOMOGENEOUS AND SECTOR HARMONICS}

Harmonic homogeneous polynomials and sector harmonics are listed below in Tables VII and VIII respectively.

TABLE VII. Harmonic homogeneous polynomials in two variables.

\begin{tabular}{lcc}
\hline \hline$n$ & $\mathcal{A}_{n}$ & $\mathcal{B}_{n}$ \\
\hline 0 & 1 & 0 \\
1 & $x$ & $y$ \\
2 & $x^{2}-y^{2}$ & $2 x y$ \\
3 & $x^{3}-3 x y^{2}$ & $3 x^{2} y-y^{3}$ \\
4 & $x^{4}-6 x^{2} y^{2}+y^{4}$ & $4 x^{3} y-4 x y^{3}$ \\
5 & $x^{5}-10 x^{3} y^{2}+5 x y^{4}$ & $5 x^{4} y-10 x^{2} y^{3}+y^{5}$ \\
6 & $x^{6}-15 x^{4} y^{2}+15 x^{2} y^{4}-y^{6}$ & $6 x^{5} y-20 x^{3} y^{3}+6 x y^{5}$ \\
7 & $x^{7}-21 x^{5} y^{2}+35 x^{3} y^{4}-7 x y^{6}$ & $7 x^{6} y-35 x^{4} y^{3}+21 x^{2} y^{5}-y^{7}$ \\
8 & $x^{8}-28 x^{6} y^{2}+70 x^{5} y^{4}-84 x^{3} y^{6}+9 x y^{8}$ & $8 x^{7} y-56 x^{5} y^{3}+56 x^{3} y^{5}-8 x y^{7}$ \\
9 & $x^{9}-36 x^{7} y^{2}+126 x^{5} y^{4}-84 x^{3} y^{6}+9 x y^{8}$ & $9 x^{8} y-84 x^{6} y^{3}+126 x^{4} y^{5}-36 x^{2} y^{7}+y^{9}$ \\
\hline \hline
\end{tabular}


TABLE VIII. Sector harmonics.

\begin{tabular}{|c|c|c|}
\hline & $n$ & \\
\hline \multirow[t]{6}{*}{$\mathcal{A}_{n}^{(\mathrm{e})}$} & 0 & 1 \\
\hline & 1 & $\ln \rho$ \\
\hline & 2 & {$\left[\frac{\rho^{2}-1}{2}-y^{2}\right]-\ln \rho$} \\
\hline & 3 & {$\left[-3 \frac{\rho^{2}-1}{2}\right]+3\left(\frac{\rho^{2}+1}{2}-y^{2}\right) \ln \rho$} \\
\hline & 4 & {$\left[\frac{3\left(\rho^{4}+4 \rho^{2}-5\right)}{8}-6 \frac{\rho^{2}-1}{2} y^{2}+y^{4}\right]-3\left(\frac{1}{2}+\rho^{2}-2 y^{2}\right) \ln \rho$} \\
\hline & 5 & {$\left[-5 \frac{9\left(\rho^{4}-1\right)}{16}+5 \times 6 \frac{\rho^{2}-1}{2} y^{2}\right]+5\left(\frac{3\left(\rho^{4}+4 \rho^{2}+1\right)}{8}-6 \frac{\rho^{2}+1}{2} y^{2}+y^{4}\right) \ln \rho$} \\
\hline \multirow[t]{6}{*}{$\mathcal{A}_{n}^{(\mathrm{m})}$} & 0 & $\frac{1}{\rho}\{1\}$ \\
\hline & 1 & $\frac{1}{\rho}\left\{\left[\frac{\rho^{2}-1}{2}\right]\right\}$ \\
\hline & 2 & $\frac{1}{\rho}\left\{\left[-\frac{\rho^{2}-1}{2}-y^{2}\right]+\rho^{2} \ln \rho\right\}$ \\
\hline & 3 & $\frac{1}{\rho}\left\{\left[\frac{3\left(\rho^{2}+1\right)}{4} \frac{\rho^{2}-1}{2}-3 \frac{\rho^{2}-1}{2} y^{2}\right]-\frac{3}{2} \rho^{2} \ln \rho\right\}$ \\
\hline & 4 & $\frac{1}{\rho}\left\{\left[-\frac{3\left(5 \rho^{4}-4 \rho^{2}-1\right)}{8}+6 \frac{\rho^{2}-1}{2} y^{2}+y^{4}\right]+\frac{3\left(2+\rho^{2}-4 y^{2}\right)}{2} \rho^{2} \ln \rho\right\}$ \\
\hline & 5 & $\frac{1}{\rho}\left\{\left[\frac{5\left(\rho^{4}+10 \rho^{2}+1\right)}{8} \frac{\rho^{2}-1}{2}-10 \frac{3\left(\rho^{2}+1\right)}{4} \frac{\rho^{2}-1}{2} y^{2}+5 \frac{\rho^{2}-1}{2} y^{4}\right]-\frac{15\left(1+\rho^{2}-4 y^{2}\right)}{4} \rho^{2} \ln \rho\right\}$ \\
\hline \multirow[t]{6}{*}{$\mathcal{B}_{n}^{(\mathrm{e})}$} & 0 & 0 \\
\hline & 1 & 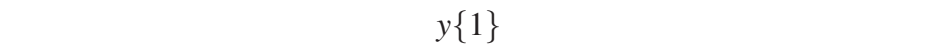 \\
\hline & 2 & $y\{2 \ln \rho\}$ \\
\hline & 3 & $y\left\{\left[3 \frac{\rho^{2}-1}{2}-y^{2}\right]-3 \ln \rho\right\}$ \\
\hline & 4 & $y\left\{\left[-12 \frac{\rho^{2}-1}{2}\right]+4\left(3 \frac{\rho^{2}+1}{2}-y^{2}\right) \ln \rho\right\}$ \\
\hline & 5 & $y\left\{\left[5 \frac{3\left(\rho^{4}+4 \rho^{2}-5\right)}{8}-10 \frac{\rho^{2}-1}{2} y^{2}+y^{4}\right]-5\left(\frac{3}{2}+3 \rho^{2}-2 y^{2}\right) \ln \rho\right\}$ \\
\hline \multirow[t]{6}{*}{$\mathcal{B}_{n}^{(\mathrm{m})}$} & 0 & 0 \\
\hline & 1 & $\frac{y}{\rho}\{1\}$ \\
\hline & 2 & $\frac{y}{\rho}\left\{\left[2 \frac{\rho^{2}-1}{2}\right]\right\}$ \\
\hline & 3 & $\frac{y}{\rho}\left\{\left[-3 \frac{\rho^{2}-1}{2}-y^{2}\right]+3 \rho^{2} \ln \rho\right\}$ \\
\hline & 4 & $\frac{y}{\rho}\left\{\left[4 \frac{3\left(\rho^{2}+1\right)}{4} \frac{\rho^{2}-1}{2}-4 \frac{\rho^{2}-1}{2} y^{2}\right]-6 \rho^{2} \ln \rho\right\}$ \\
\hline & 5 & $\frac{y}{\rho}\left\{\left[-5 \frac{3\left(5 \rho^{4}-4 \rho^{2}-1\right)}{8}+10 \frac{\rho^{2}-1}{2} y^{2}+y^{4}\right]+5\left(3+\frac{3}{2} \rho^{2}-2 y^{2}\right) \rho^{2} \ln \rho\right\}$ \\
\hline
\end{tabular}




\section{APPENDIX E: R- AND S-MULTIPOLES-EXACT EXPRESSIONS}

The scalar potentials, longitudinal component of vector potential, and field components for pure R-and S-multipoles up to fifth order are listed in Tables IX and X and Tables XI and XII, respectively.

TABLE IX. Longitudinal component of the vector potential and scalar potential for pure normal and skew R-multipoles.

\begin{tabular}{|c|c|c|c|}
\hline$n$ & & $A_{z}$ & $\Phi$ \\
\hline 0 & Calibration & $-\bar{C}_{0}$ & 0 \\
\hline 1 & Normal dipole & $-\frac{1}{1 !}(x) \bar{C}_{1}$ & $-\frac{1}{1 !}(y) \bar{C}_{1}$ \\
\hline 2 & Normal quadrupole & $-\frac{1}{2 !}\left(x^{2}-y^{2}\right) \bar{C}_{2}$ & $-\frac{1}{2 !}(2 x y) \bar{C}_{2}$ \\
\hline 3 & Normal sextupole & $-\frac{1}{3 !}\left(x^{3}-3 x y^{2}\right) \bar{C}_{3}$ & $-\frac{1}{3 !}\left(3 x^{2} y-y^{3}\right) \bar{C}_{3}$ \\
\hline 4 & Normal octupole & $-\frac{1}{4 !}\left(x^{4}-6 x^{2} y^{2}+y^{4}\right) \bar{C}_{4}$ & $-\frac{1}{4 !}\left(4 x^{3} y-4 x y^{3}\right) \bar{C}_{4}$ \\
\hline 5 & Normal decapole & $-\frac{1}{5 !}\left(x^{5}-10 x^{3} y^{2}+5 x y^{4}\right) \bar{C}_{5}$ & $-\frac{1}{5 !}\left(5 x^{4} y-10 x^{2} y^{3}+y^{5}\right) \bar{C}_{5}$ \\
\hline 0 & Calibration & 0 & $\underline{C}_{0}$ \\
\hline 1 & Skew dipole & $\frac{1}{1 !}(y) \underline{C}_{1}$ & $-\frac{1}{1 !}(x) \underline{C}_{1}$ \\
\hline 2 & Skew quadrupole & $\frac{1}{2 !}(2 x y) \underline{C}_{2}$ & $-\frac{1}{2 !}\left(x^{2}-y^{2}\right) \underline{C}_{2}$ \\
\hline 3 & Skew sextupole & $\frac{1}{3 !}\left(3 x^{2} y-y^{3}\right) \underline{C}_{3}$ & $-\frac{1}{3 !}\left(x^{3}-3 x y^{2}\right) \underline{C}_{3}$ \\
\hline 4 & Skew octupole & $\frac{1}{4 !}\left(4 x^{3} y-4 x y^{3}\right) \underline{C}_{4}$ & $-\frac{1}{4 !}\left(x^{4}-6 x^{2} y^{2}+y^{4}\right) \underline{C}_{4}$ \\
\hline 5 & Skew decapole & $\frac{1}{5 !}\left(5 x^{4} y-10 x^{2} y^{3}+y^{5}\right) \underline{C}_{5}$ & $-\frac{1}{5 !}\left(x^{5}-10 x^{3} y^{2}+5 x y^{4}\right) \underline{C}_{5}$ \\
\hline
\end{tabular}

TABLE X. Horizontal and vertical components of pure normal and skew R-multipole magnets' field.

\begin{tabular}{|c|c|c|c|}
\hline$n$ & & $F_{x}$ & $F_{y}$ \\
\hline 0 & Calibration & $\ldots$ & $\ldots$ \\
\hline 1 & Normal dipole & 0 & $\bar{C}_{1}$ \\
\hline 2 & Normal quadrupole & $\frac{1}{1 !}(y) \bar{C}_{2}$ & $\frac{1}{1 !}(x) \bar{C}_{2}$ \\
\hline 3 & Normal sextupole & $\frac{1}{2 !}(2 x y) \bar{C}_{3}$ & $\frac{1}{2 !}\left(x^{2}-y^{2}\right) \bar{C}_{3}$ \\
\hline 4 & Normal octupole & $\frac{1}{3 !}\left(3 x^{2} y-y^{3}\right) \bar{C}_{4}$ & $\frac{1}{3 !}\left(x^{3}-3 x y^{2}\right) \bar{C}_{4}$ \\
\hline 5 & Normal decapole & $\frac{1}{4 !}\left(4 x^{3} y-4 x y^{3}\right) \bar{C}_{5}$ & $\frac{1}{4 !}\left(x^{4}-6 x^{2} y^{2}+y^{4}\right) \bar{C}_{5}$ \\
\hline 0 & Calibration & $\ldots$ & $\cdots$ \\
\hline 1 & Skew dipole & $\underline{C}_{1}$ & 0 \\
\hline 2 & Skew quadrupole & $\frac{1}{1 !}(x) \underline{C}_{2}$ & $-\frac{1}{1 !}(y) \underline{C}_{2}$ \\
\hline 3 & Skew sextupole & $\frac{1}{2 !}\left(x^{2}-y^{2}\right) \underline{C}_{3}$ & $-\frac{1}{2 !}(2 x y) \underline{C}_{3}$ \\
\hline 4 & Skew octupole & $\frac{1}{3 !}\left(x^{3}-3 x y^{2}\right) \underline{C}_{4}$ & $-\frac{1}{3 !}\left(3 x^{2} y-y^{3}\right) \underline{C}_{4}$ \\
\hline 5 & Skew decapole & $\frac{1}{4 !}\left(x^{4}-6 x^{2} y^{2}+y^{4}\right) \underline{C}_{5}$ & $-\frac{1}{4 !}\left(4 x^{3} y-4 x y^{3}\right) \underline{C}_{5}$ \\
\hline
\end{tabular}


TABLE XI. Azimuthal component of the vector potential and scalar potential for pure normal and skew S-multipoles.

\begin{tabular}{|c|c|c|}
\hline & $n$ & \\
\hline \multirow[t]{6}{*}{$\bar{A}_{\theta}^{(n)}$} & 0 & $-\frac{1}{0 !} \frac{1}{\rho}\{1\} \bar{C}_{0}$ \\
\hline & 1 & $-\frac{1}{1 !} \frac{1}{\rho}\left\{\left[\frac{\rho^{2}-1}{2}\right]\right\} \bar{C}_{1}$ \\
\hline & 2 & $-\frac{1}{2 !} \frac{1}{\rho}\left\{\left[-\frac{\rho^{2}-1}{2}-y^{2}\right]+\rho^{2} \ln \rho\right\} \bar{C}_{2}$ \\
\hline & 3 & $-\frac{1}{3 !} \frac{1}{\rho}\left\{\left[\frac{3\left(\rho^{2}+1\right)}{4} \frac{\rho^{2}-1}{2}-3 \frac{\rho^{2}-1}{2} y^{2}\right]-\frac{3}{2} \rho^{2} \ln \rho\right\} \bar{C}_{3}$ \\
\hline & 4 & $-\frac{1}{4 !} \frac{1}{\rho}\left\{\left[-\frac{3\left(5 \rho^{4}-4 \rho^{2}-1\right)}{8}+6 \frac{\rho^{2}-1}{2} y^{2}+y^{4}\right]+\frac{3\left(2+\rho^{2}-4 y^{2}\right)}{2} \rho^{2} \ln \rho\right\} \bar{C}_{4}$ \\
\hline & 5 & $-\frac{1}{5 !} \frac{1}{\rho}\left\{\left[\frac{5\left(\rho^{4}+10 \rho^{2}+1\right)}{8} \frac{\rho^{2}-1}{2}-10 \frac{3\left(\rho^{2}+1\right)}{4} \frac{\rho^{2}-1}{2} y^{2}+5 \frac{\rho^{2}-1}{2} y^{4}\right]-\frac{15\left(1+\rho^{2}-4 y^{2}\right)}{4} \rho^{2} \ln \rho\right\} \bar{C}_{5}$ \\
\hline \multirow[t]{6}{*}{$\bar{\Phi}^{(n)}$} & 0 & 0 \\
\hline & 1 & $-\frac{1}{1 !} y\{1\} \bar{C}_{1}$ \\
\hline & 2 & $-\frac{1}{2 !} y\{2 \ln \rho\} \bar{C}_{2}$ \\
\hline & 3 & $-\frac{1}{3 !} y\left\{\left[3 \frac{\rho^{2}-1}{2}-y^{2}\right]-3 \ln \rho\right\} \bar{C}_{3}$ \\
\hline & 4 & $-\frac{1}{4 !} y\left\{\left[-12 \frac{\rho^{2}-1}{2}\right]+4\left(3 \frac{\rho^{2}+1}{2}-y^{2}\right) \ln \rho\right\} \bar{C}_{4}$ \\
\hline & 5 & $-\frac{1}{5 !} y\left\{\left[5 \frac{3\left(\rho^{4}+4 \rho^{2}-5\right)}{8}-10 \frac{\rho^{2}-1}{2} y^{2}+y^{4}\right]-5\left(\frac{3}{2}+3 \rho^{2}-2 y^{2}\right) \ln \rho\right\} \bar{C}_{5}$ \\
\hline \multirow[t]{6}{*}{$\underline{A}_{\theta}^{(n)}$} & 0 & 0 \\
\hline & 1 & $\frac{1}{1 !} \frac{y}{\rho}\{1\} \underline{C}_{1}$ \\
\hline & 2 & 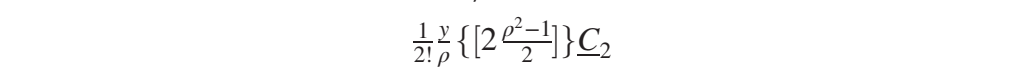 \\
\hline & 3 & $\frac{1}{3 !} \frac{y}{\rho}\left\{\left[-3 \frac{\rho^{2}-1}{2}-y^{2}\right]+3 \rho^{2} \ln \rho\right\} \underline{C}_{3}$ \\
\hline & 4 & $\frac{1}{4 !} \frac{y}{\rho}\left\{\left[4 \frac{3\left(\rho^{2}+1\right)}{4} \frac{\rho^{2}-1}{2}-4 \frac{\rho^{2}-1}{2} y^{2}\right]-6 \rho^{2} \ln \rho\right\} \underline{C}_{4}$ \\
\hline & 5 & $\frac{1}{5 !} \underline{y}\left\{\left[-5 \frac{3\left(5 \rho^{4}-4 \rho^{2}-1\right)}{8}+10 \frac{\rho^{2}-1}{2} y^{2}+y^{4}\right]+5\left(3+\frac{3}{2} \rho^{2}-2 y^{2}\right) \rho^{2} \ln \rho\right\} C_{5}$ \\
\hline \multirow[t]{6}{*}{$\underline{\Phi}^{(n)}$} & 0 & $-\frac{1}{0 !}\{1\} \underline{C}_{0}$ \\
\hline & 1 & $-\frac{1}{1 !}\{\ln \rho\} \underline{C}_{1}$ \\
\hline & 2 & $-\frac{1}{2 !}\left\{\left[\frac{\rho^{2}-1}{2}-y^{2}\right]-\ln \rho\right\} \underline{C_{2}}$ \\
\hline & 3 & $-\frac{1}{3 !}\left\{\left[-3 \frac{\rho^{2}-1}{2}\right]+3\left(\frac{\rho^{2}+1}{2}-y^{2}\right) \ln \rho\right\} \underline{C}_{3}$ \\
\hline & 4 & $-\frac{1}{4 !}\left\{\left[\frac{3\left(\rho^{4}+4 \rho^{2}-5\right)}{8}-6 \frac{\rho^{2}-1}{2} y^{2}+y^{4}\right]-3\left(\frac{1}{2}+\rho^{2}-2 y^{2}\right) \ln \rho\right\} \underline{C}_{4}$ \\
\hline & 5 & $-\frac{1}{5 !}\left\{\left[-5 \frac{9\left(\rho^{4}-1\right)}{16}+5 \times 6 \frac{\rho^{2}-1}{2} y^{2}\right]+5\left(\frac{3\left(\rho^{4}+4 \rho^{2}+1\right)}{8}-6 \frac{\rho^{2}+1}{2} y^{2}+y^{4}\right) \ln \rho\right\} \underline{C}_{5}$ \\
\hline
\end{tabular}


TABLE XII. Radial and vertical components of pure normal and skew S-multipoles' field.

\begin{tabular}{|c|c|c|c|}
\hline & $n$ & & \\
\hline \multirow[t]{6}{*}{$\bar{F}_{\rho}^{(n)}$} & 0 & Calibration & $\cdots$ \\
\hline & 1 & Normal dipole & 0 \\
\hline & 2 & Normal quadrupole & $-\frac{1}{1 !} \frac{y}{\rho}\{1\} \bar{C}_{2}$ \\
\hline & 3 & Normal sextupole & $-\frac{1}{2 !} \frac{y}{\rho}\left\{\left[2 \frac{\rho^{2}-1}{2}\right]\right\} \bar{C}_{3}$ \\
\hline & 4 & Normal octupole & $-\frac{1}{3 !} \frac{y}{\rho}\left\{\left[-3 \frac{\rho^{2}-1}{2}-y^{2}\right]+3 \rho^{2} \ln \rho\right\} \bar{C}_{4}$ \\
\hline & 5 & Normal decapole & $-\frac{1}{4 !} \frac{y}{\rho}\left\{\left[4 \frac{3\left(\rho^{2}+1\right)}{4} \frac{\rho^{2}-1}{2}-4 \frac{\rho^{2}-1}{2} y^{2}\right]-6 \rho^{2} \ln \rho\right\} \bar{C}_{5}$ \\
\hline \multirow[t]{6}{*}{$\bar{F}_{\mathrm{y}}^{(n)}$} & 0 & Calibration & $\cdots$ \\
\hline & 1 & Normal dipole & $\frac{1}{0 !}\{1\} \bar{C}_{1}$ \\
\hline & 2 & Normal quadrupole & $\frac{1}{1 !}\{\ln \rho\} \bar{C}_{2}$ \\
\hline & 3 & Normal sextupole & $\frac{1}{2 !}\left\{\left[\frac{\rho^{2}-1}{2}-y^{2}\right]-\ln \rho\right\} \bar{C}_{3}$ \\
\hline & 4 & Normal octupole & $\frac{1}{3 !}\left\{\left[-3 \frac{\rho^{2}-1}{2}\right]+3\left(\frac{\rho^{2}+1}{2}-y^{2}\right) \ln \rho\right\} \bar{C}_{4}$ \\
\hline & 5 & Normal decapole & $\frac{1}{4 !}\left\{\left[\frac{3\left(\rho^{4}+4 \rho^{2}-5\right)}{8}-6 \frac{\rho^{2}-1}{2} y^{2}+y^{4}\right]-3\left(\frac{1}{2}+\rho^{2}-2 y^{2}\right) \ln \rho\right\} \bar{C}_{5}$ \\
\hline \multirow[t]{6}{*}{$\underline{F}_{\rho}^{(n)}$} & 0 & Calibration & $\cdots$ \\
\hline & 1 & Skew dipole & $\frac{1}{0 !} \frac{1}{\rho}\{1\} \underline{C}_{1}$ \\
\hline & 2 & Skew quadrupole & $\frac{1}{1 !} \frac{1}{\rho}\left\{\left[\frac{\rho^{2}-1}{2}\right]\right\} \underline{C}_{2}$ \\
\hline & 3 & Skew sextupole & $\frac{1}{2 !} \frac{1}{\rho}\left\{\left[-\frac{\rho^{2}-1}{2}-y^{2}\right]+\rho^{2} \ln \rho\right\} \underline{C}_{3}$ \\
\hline & 4 & Skew octupole & $\frac{1}{3 !} \frac{1}{\rho}\left\{\left[\frac{3\left(\rho^{2}+1\right)}{4} \frac{\rho^{2}-1}{2}-3 \frac{\rho^{2}-1}{2} y^{2}\right]-\frac{3}{2} \rho^{2} \ln \rho\right\} \underline{C}_{4}$ \\
\hline & 5 & Skew decapole & $\frac{1}{4 !} \frac{1}{\rho}\left\{\left[-\frac{3\left(5 \rho^{4}-4 \rho^{2}-1\right)}{8}+6 \frac{\rho^{2}-1}{2} y^{2}+y^{4}\right]+\frac{3\left(2+\rho^{2}-4 y^{2}\right)}{2} \rho^{2} \ln \rho\right\} \underline{C}_{5}$ \\
\hline \multirow[t]{6}{*}{$\underline{F}_{\mathrm{y}}^{(n)}$} & 0 & Calibration & $\cdots$ \\
\hline & 1 & Skew dipole & 0 \\
\hline & 2 & Skew quadrupole & $\frac{1}{1 !} y\{1\} \underline{C}_{2}$ \\
\hline & 3 & Skew sextupole & $\frac{1}{2 !} y\{2 \ln \rho\} \underline{C}_{3}$ \\
\hline & 4 & Skew octupole & $\frac{1}{3 !} y\left\{\left[3 \frac{\rho^{2}-1}{2}-y^{2}\right]-3 \ln \rho\right\} \underline{C}_{4}$ \\
\hline & 5 & Skew decapole & $\frac{1}{4 !} y\left\{\left[-12 \frac{\rho^{2}-1}{2}\right]+4\left(3 \frac{\rho^{2}+1}{2}-y^{2}\right) \ln \rho\right\} \underline{C}_{5}$ \\
\hline
\end{tabular}




\section{APPENDIX F: TAYLOR POLYNOMIALS OF $\mathcal{F}_{n}$ AND $\mathcal{G}_{n}$}

The first ten terms of the Maclaurin series of $\mathcal{F}_{n}(x), \mathcal{G}_{n}(x)$ and $\frac{\mathcal{G}_{n}(x)}{1+x}$ are listed in Table XIII.

TABLE XIII. Maclaurin series of $\mathcal{F}_{n}(x), \mathcal{G}_{n}(x)$ and $\frac{\mathcal{G}_{n}(x)}{1+x}$; they are also Taylor polynomials of $\mathcal{F}_{n}(\rho), \mathcal{G}_{n}(\rho)$ and $\frac{\mathcal{G}_{n}(\rho)}{\rho}$ at $\rho=1$.

\begin{tabular}{|c|c|}
\hline$n$ & $\mathrm{~T}\left(\mathcal{F}_{n}\right)$ \\
\hline 0 & 1 \\
\hline 1 & $x-\frac{1}{2} x^{2}+\frac{1}{3} x^{3}-\frac{1}{4} x^{4}+\frac{1}{5} x^{5}-\frac{1}{6} x^{6}+\frac{1}{7} x^{7}-\frac{1}{8} x^{8}+\frac{1}{9} x^{9}-\frac{1}{10} x^{10}+O\left(x^{11}\right)$ \\
\hline 2 & $x^{2}-\frac{1}{3} x^{3}+\frac{1}{4} x^{4}-\frac{1}{5} x^{5}+\frac{1}{6} x^{6}-\frac{1}{7} x^{7}+\frac{1}{8} x^{8}-\frac{1}{9} x^{9}+\frac{1}{10} x^{10}-\frac{1}{11} x^{11}+O\left(x^{12}\right)$ \\
\hline 3 & $x^{3}-\frac{1}{2} x^{4}+\frac{7}{20} x^{5}-\frac{11}{40} x^{6}+\frac{8}{35} x^{7}-\frac{11}{56} x^{8}+\frac{29}{168} x^{9}-\frac{37}{240} x^{10}+\frac{23}{165} x^{11}-\frac{7}{55} x^{12}+O\left(x^{13}\right)$ \\
\hline 4 & $x^{4}-\frac{2}{5} x^{5}+\frac{3}{10} x^{6}-\frac{17}{70} x^{7}+\frac{23}{112} x^{8}-\frac{5}{28} x^{9}+\frac{19}{120} x^{10}-\frac{47}{330} x^{11}+\frac{57}{440} x^{12}-\frac{17}{143} x^{13}+O\left(x^{14}\right)$ \\
\hline 5 & $x^{5}-\frac{1}{2} x^{6}+\frac{5}{14} x^{7}-\frac{2}{7} x^{8}+\frac{27}{112} x^{9}-\frac{47}{224} x^{10}+\frac{689}{3696} x^{11}-\frac{355}{2112} x^{12}+\frac{263}{1716} x^{13}-\frac{1129}{8008} x^{14}+O\left(x^{15}\right)$ \\
\hline 6 & $x^{6}-\frac{3}{7} x^{7}+\frac{9}{28} x^{8}-\frac{11}{42} x^{9}+\frac{25}{112} x^{10}-\frac{241}{1232} x^{11}+\frac{123}{704} x^{12}-\frac{181}{1144} x^{13}+\frac{2319}{16016} x^{14}-\frac{535}{4004} x^{15}+O\left(x^{16}\right)$ \\
\hline 7 & $x^{7}-\frac{1}{2} x^{8}+\frac{13}{36} x^{9}-\frac{7}{24} x^{10}+\frac{131}{528} x^{11}-\frac{689}{3168} x^{12}+\frac{5339}{27456} x^{13}-\frac{9683}{54912} x^{14}+\frac{16}{99} x^{15}-\frac{1367}{9152} x^{16}+O\left(x^{17}\right)$ \\
\hline 8 & $x^{8}-\frac{4}{9} x^{9}+\frac{1}{3} x^{10}-\frac{3}{11} x^{11}+\frac{185}{792} x^{12}-\frac{353}{1716} x^{13}+\frac{1267}{6864} x^{14}-\frac{3457}{20592} x^{15}+\frac{5647}{36608} x^{16}-\frac{855}{5984} x^{17}+O\left(x^{18}\right)$ \\
\hline 9 & $x^{9}-\frac{1}{2} x^{10}+\frac{4}{11} x^{11}-\frac{13}{44} x^{12}+\frac{289}{1144} x^{13}-\frac{509}{2288} x^{14}+\frac{457}{2288} x^{15}-\frac{2}{11} x^{16}+\frac{9461}{56576} x^{17}-\frac{192991}{1244672} x^{18}+O\left(x^{19}\right)$ \\
\hline$n$ & $\mathrm{~T}\left(\mathcal{G}_{n}\right)$ \\
\hline 0 & 1 \\
\hline 1 & $x+\frac{1}{2} x^{2}$ \\
\hline 2 & $x^{2}+\frac{1}{3} x^{3}-\frac{1}{12} x^{4}+\frac{1}{30} x^{5}-\frac{1}{60} x^{6}+\frac{1}{105} x^{7}-\frac{1}{168} x^{8}+\frac{1}{252} x^{9}-\frac{1}{360} x^{10}+\frac{1}{495} x^{11}+O\left(x^{12}\right)$ \\
\hline 3 & $x^{3}+\frac{1}{2} x^{4}-\frac{1}{20} x^{5}+\frac{1}{40} x^{6}-\frac{1}{70} x^{7}+\frac{1}{112} x^{8}-\frac{1}{168} x^{9}+\frac{1}{240} x^{10}-\frac{1}{330} x^{11}+\frac{1}{440} x^{12}+O\left(x^{13}\right)$ \\
\hline 4 & $x^{4}+\frac{2}{5} x^{5}-\frac{1}{10} x^{6}+\frac{3}{70} x^{7}-\frac{13}{560} x^{8}+\frac{1}{70} x^{9}-\frac{1}{105} x^{10}+\frac{31}{4620} x^{11}-\frac{13}{2640} x^{12}+\frac{8}{2145} x^{13}+O\left(x^{14}\right)$ \\
\hline 5 & $x^{5}+\frac{1}{2} x^{6}-\frac{1}{14} x^{7}+\frac{1}{28} x^{8}-\frac{1}{48} x^{9}+\frac{3}{224} x^{10}-\frac{17}{1848} x^{11}+\frac{7}{1056} x^{12}-\frac{17}{3432} x^{13}+\frac{61}{16016} x^{14}+O\left(x^{15}\right)$ \\
\hline 6 & $x^{6}+\frac{3}{7} x^{7}-\frac{3}{28} x^{8}+\frac{1}{21} x^{9}-\frac{3}{112} x^{10}+\frac{3}{176} x^{11}-\frac{173}{14784} x^{12}+\frac{271}{32032} x^{13}-\frac{37}{5824} x^{14}+\frac{59}{12012} x^{15}+O\left(x^{16}\right)$ \\
\hline 7 & $x^{7}+\frac{1}{2} x^{8}-\frac{1}{12} x^{9}+\frac{1}{24} x^{10}-\frac{13}{528} x^{11}+\frac{17}{1056} x^{12}-\frac{103}{1952} x^{13}+\frac{151}{18304} x^{14}-\frac{43}{6864} x^{15}+\frac{179}{36609} x^{16}+O\left(x^{17}\right)$ \\
\hline 8 & $x^{8}+\frac{4}{9} x^{9}-\frac{1}{9} x^{10}+\frac{5}{99} x^{11}-\frac{23}{792} x^{12}+\frac{97}{5148} x^{13}-\frac{271}{20592} x^{14}+\frac{199}{20592} x^{15}-\frac{2425}{329472} x^{16}+\frac{1009}{175032} x^{17}+O\left(x^{18}\right)$ \\
\hline 9 & $x^{9}+\frac{1}{2} x^{10}-\frac{1}{11} x^{11}+\frac{1}{22} x^{12}-\frac{31}{1144} x^{13}+\frac{41}{2288} x^{14}-\frac{29}{2288} x^{15}+\frac{43}{4576} x^{16}-\frac{4489}{622336} x^{17}+\frac{7079}{1244672} x^{18}+O\left(x^{19}\right)$ \\
\hline$n$ & $\mathrm{~T}\left(\mathcal{G}_{n} / \rho\right)$ \\
\hline 0 & $1-x+x^{2}-x^{3}+x^{4}-x^{5}+x^{6}-x^{7}+x^{8}-x^{9}+O\left(x^{10}\right)$ \\
\hline 1 & $x-\frac{1}{2} x^{2}+\frac{1}{2} x^{3}-\frac{1}{2} x^{4}+\frac{1}{2} x^{5}-\frac{1}{2} x^{6}+\frac{1}{2} x^{7}-\frac{1}{2} x^{8}+\frac{1}{2} x^{9}-\frac{1}{2} x^{10}+O\left(x^{11}\right)$ \\
\hline 2 & $x^{2}-\frac{2}{3} x^{3}+\frac{7}{12} x^{4}-\frac{11}{20} x^{5}+\frac{8}{15} x^{6}-\frac{11}{21} x^{7}+\frac{29}{56} x^{8}-\frac{37}{72} x^{9}+\frac{23}{45} x^{10}-\frac{28}{55} x^{11}+O\left(x^{12}\right)$ \\
\hline 3 & $x^{3}-\frac{1}{2} x^{4}+\frac{9}{20} x^{5}-\frac{17}{40} x^{6}+\frac{23}{56} x^{7}-\frac{45}{112} x^{8}+\frac{19}{48} x^{9}-\frac{47}{120} x^{10}+\frac{171}{440} x^{11}-\frac{17}{44} x^{12}+O\left(x^{13}\right)$ \\
\hline 4 & $x^{4}-\frac{3}{5} x^{5}+\frac{1}{2} x^{6}-\frac{16}{35} x^{7}+\frac{243}{560} x^{8}-\frac{47}{112} x^{9}+\frac{689}{1680} x^{10}-\frac{71}{176} x^{11}+\frac{263}{660} x^{12}-\frac{1129}{2860} x^{13}+O\left(x^{14}\right)$ \\
\hline 5 & $x^{5}-\frac{1}{2} x^{6}+\frac{3}{7} x^{7}-\frac{11}{28} x^{8}+\frac{125}{336} x^{9}-\frac{241}{672} x^{10}+\frac{123}{352} x^{11}-\frac{181}{528} x^{12}+\frac{773}{2288} x^{13}-\frac{2675}{8008} x^{14}+O\left(x^{15}\right)$ \\
\hline 6 & $x^{6}-\frac{4}{7} x^{7}+\frac{13}{28} x^{8}-\frac{5}{12} x^{9}+\frac{131}{336} x^{10}-\frac{689}{1848} x^{11}+\frac{5339}{14784} x^{12}-\frac{9683}{27456} x^{13}+\frac{80}{231} x^{14}-\frac{1367}{4004} x^{15}+O\left(x^{16}\right)$ \\
\hline 7 & $x^{7}-\frac{1}{2} x^{8}+\frac{5}{12} x^{9}-\frac{3}{8} x^{10}+\frac{185}{528} x^{11}-\frac{353}{1056} x^{12}+\frac{8869}{27456} x^{13}-\frac{17285}{54912} x^{14}+\frac{5647}{18304} x^{15}-\frac{855}{2816} x^{16}+O\left(x^{17}\right)$ \\
\hline 8 & $x^{8}-\frac{5}{9} x^{9}+\frac{4}{9} x^{10}-\frac{13}{33} x^{11}+\frac{289}{792} x^{12}-\frac{3563}{10296} x^{13}+\frac{2285}{6864} x^{14}-\frac{32}{99} x^{15}+\frac{9461}{29952} x^{16}-\frac{192991}{622336} x^{17}+O\left(x^{18}\right)$ \\
\hline 9 & $x^{9}-\frac{1}{2} x^{10}+\frac{9}{22} x^{11}-\frac{4}{11} x^{12}+\frac{35}{104} x^{13}-\frac{729}{2288} x^{14}+\frac{175}{572} x^{15}-\frac{1357}{4576} x^{16}+\frac{13851}{47872} x^{17}-\frac{353047}{1244672} x^{18}+O\left(x^{19}\right)$ \\
\hline
\end{tabular}


[1] K. L. Brown, SLAC Report No. SLAC-75, 1972, http://cds .cern.ch/record/283218/files/SLAC-75.pdf.

[2] E. Forest, Beam Dynamics (Harwood Academic Publishers, Amsterdam, 1998), Vol. 8.

[3] H. Wiedemann, Particle Accelerator Physics (Springer, New York, 2015).

[4] E. M. McMillan, Multipoles in cylindrical coordinates, Nucl. Instrum. Methods 127, 471 (1975).
[5] S. Mane, Solutions of Laplace's equation in two dimensions with a curved longitudinal axis, Nucl. Instrum. Methods Phys. Res., Sect. A 321, 365 (1992).

[6] R. A. Beth, Complex representation and computation of two-dimensional magnetic fields, J. Appl. Phys. 37, 2568 (1966).

[7] H. Yoshida, Construction of higher order symplectic integrators, Phys. Lett. A 150, 262 (1990). 